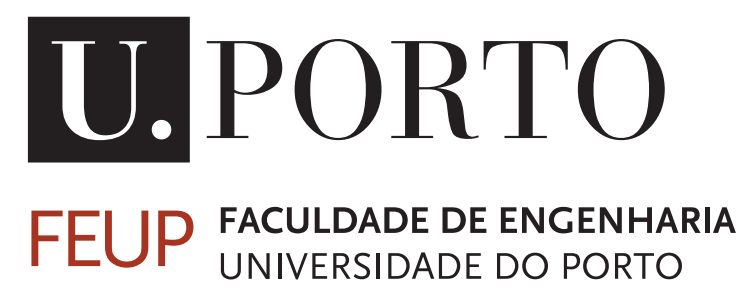

\title{
Automation of Waste Sorting with Deep Learning
}

\author{
João Soares Sousa
}

Mestrado Integrado em Engenharia Eletrotécnica e de Computadores

Supervisor: Professor Doutor Jaime S. Cardoso

Second Supervisor: Professora Doutora Ana Rebelo

July 22, 2019 
(C) João Sousa, 2019 


\section{Resumo}

A importância da reciclagem é bem conhecida, seja por razões ambientais ou economicas, é impossível escapar-lhe e a indústria exige eficiência. O trabalho manual e as técnicas industiais tradicionais de separação de lixo não são capazes de atingir os objetivos exigidos pela comunidade internacional. Soluções baseadas em técnicas de visão computacional têm o potencial de automatizar parte das tarefas de manuseio de resíduos.

Nesta dissertação, é proposta uma abordagem hierárquica baseada em deep learning para detecção e classificação de resíduos em tabuleiros de refeições. Esta abordagem em duas etapas mantém as vantagens dos algoritmos de deteção de objetos recentes (como por exemplo da Faster R-CNN) e permite que a tarefa de classificação seja suportada por caixas delimitadoras (bounding boxes) de resolução mais alta. Além disso, também foi adquirida, anotada e disponibilizada para a comunidade científica uma nova base de dados, denomindada de Labeled Waste in the Wild, para fins de pesquisa. Na comparação experimental com abordagens padrão de deep learning, o modelo hierárquico proposto apresenta melhor desempenho de detecção e classificação. 


\section{Abstract}

The importance of recycling is well known, either for environmental or economic reasons, it is impossible to escape it and the industry demands efficiency. Manual labour and traditional industrial sorting techniques are not capable of keeping up with the objectives demanded by the international community. Solutions based in computer vision techniques have the potential automate part of the waste handling tasks.

In this dissertation, a hierarchical deep learning approach for waste detection and classification in food trays is proposed. The proposed two-step approach retains the advantages of recent object detectors (as Faster R-CNN) and allows the classification task to be supported in higher resolution bounding boxes. Additionally, it was also collected, annotated and made available to the scientific community a new dataset, named Labeled Waste in the Wild, for research and benchmark purposes. In the experimental comparison with standard deep learning approaches, the proposed hierarchical model shows better detection and classification performance. 


\section{Agradecimentos}

Gostaria de expressar a minha sincera gratidão ao professor Jaime Cardoso, pela disponibilidade, e pelas suas ideias que elevaram este projeto. Também com a ajuda da professora Ana Rebelo, melhorei como estudante e engenheiro. Uma palavra ao INESC TEC e ao membros do CTM por me terem acolhido e ajudado sempre que necessário.

Porque esta tese é também fruto dos últimos cinco anos, gostaria de agradecer ao professor Armando Sousa pelas experiências fantasticas e enriquecedoras na Universidade Júnior, Mostra UP e SPE. Aos amigos dos mortais encarpados, pelos trabalhos e principalmentes pelas pausas que fizemos juntos, aos que comigo escaparam civil para o mundo das eletrónicas, e aqueles com quem partilhei A aventura do outro lado do mundo, o meu sincero obrigado.

Aos amigos de Sobrado, aos primos e família, que sempre tentaram que tivesse contacto com o mundo real, mesmo quando me fechava na FEUP. À Natacha, por toda a paciência nas ausências, pela ajuda técnica, por me ouvir mesmo quando nada percebia. Por toda a motivação e apoio, esta tese também é tua.

Por fim, agradecer aos meus pais, por terem sido os melhores exemplos que poderia ter, pela educação, pelas viagens e oportunidades. O que sou hoje a eles lhes devo.

João Soares Sousa 
"Now this is not the end. It is not even the beginning of the end. But it is, perhaps, the end of the beginning."

Winston Churchill 


\section{Contents}

1 Introduction 1

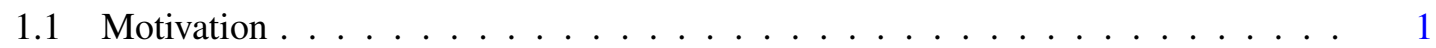

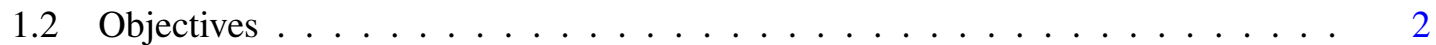

1.3 Dissertation Structure . . . . . . . . . . . . . . . . . . 3

2 State Of The Art 5

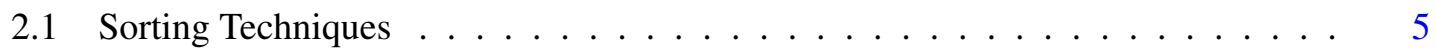

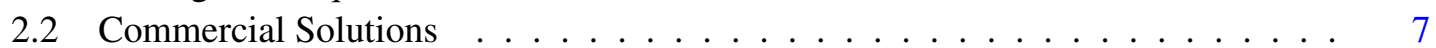

2.3 Related Work . . . . . . . . . . . . . . . . . . . . 8

2.3.1 State-of-the-Art solutions in waste classification . . . . . . . . . . 9

2.4 Background Knowledge . . . . . . . . . . . . . . . . . . . . . . 10

2.4.1 Convolutional Neural Network . . . . . . . . . . . . . . . . . . . 10

2.5 State-of-the-Art in visual object detection algorithms . . . . . . . . . . . . . 13

$2.5 .1 \quad \mathrm{R}-\mathrm{CNN} \ldots \ldots \ldots \ldots \ldots$

2.5 .2 Fast R-CNN . . . . . . . . . . . . . . . . . . . . . . . . 15

2.5 .3 Faster R-CNN . . . . . . . . . . . . . . . . . . . . . . . 17

2.5 .4 YOLO (You Only Look Once) . . . . . . . . . . . . . . . . . 20

2.5 .5 RetinaNet . . . . . . . . . . . . . . . . . 22

3 Labeled Waste in the Wild Dataset 27

3.1 Classes and Multi-label perspective . . . . . . . . . . . . . . . 28

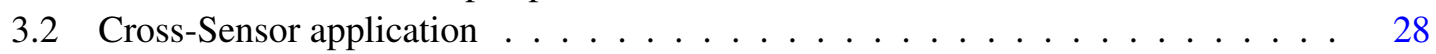

3.3 Summary . . . . . . . . . . . . . . . . . . . . . 29

4 Hierarchical Deep Detection and Classification of Waste 31

4.1 Standard baseline approach: Flat method . . . . . . . . . . . . . . . . 31

4.2 Improved baseline approach: Cascaded Region Proposal and Classification . . . 32

$4.2 .1 \quad$ CNN Development . . . . . . . . . . . . . . . . . . . . . 32

4.3 Hierarchical approach . . . . . . . . . . . . . . . . . 34

4.3.1 Material based method . . . . . . . . . . . . . . . . . . 34

4.3.2 Shape based method . . . . . . . . . . . . . . . 35

5 Experimental Study 37

5.1 Metrics . . . . . . . . . . . . . . . . . . . . . . . 37

5.1 .1 Intersection over Union . . . . . . . . . . . . . . . . . . . 37

5.1 .2 Average Precision . . . . . . . . . . . . . . . . 38

5.2 Standard baseline approach - Flat method . . . . . . . . . . . . . . . . 39

5.3 Cascaded Region Proposal and Classification . . . . . . . . . . . . . . . . . 39 
$5.3 .1 \quad \mathrm{CNN}$ performance $\ldots \ldots \ldots \ldots \ldots$

5.3 .2 Integrated model performance . . . . . . . . . . . . . 41

5.4 Hierarchical Model . . . . . . . . . . . . . . . . . . . . . . . . . . . . 41

5.4 Material Based . . . . . . . . . . . . . . . . . . 41

5.4 .2 Shape Based . . . . . . . . . . . . . . . . . . 42

5.5 Cross-Sensor Validation . . . . . . . . . . . . . . . . . . . . . . 43

5.6 Discussion . . . . . . . . . . . . . . . . . . . . . . . 44

5.6.1 Model Performance Overview . . . . . . . . . . . . . . . . . 44

5.6.2 Influence of Less Represented Classes . . . . . . . . . . . . . . . . . . . . . 45

5.6.3 Understanding Cross-Sensor Validation . . . . . . . . . . . . . . . . 46

6 Conclusions 49

6.1 Research Objectives . . . . . . . . . . . . . . . . . . . . . . . . . . . . . . 49

6.2 Strengths and Weaknesses . . . . . . . . . . . . . . . . . . 50

6.3 Future Work . . . . . . . . . . . . . . . . . . . 50

A Detection Examples

References $\quad 55$ 


\section{List of Figures}

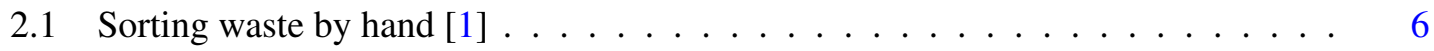

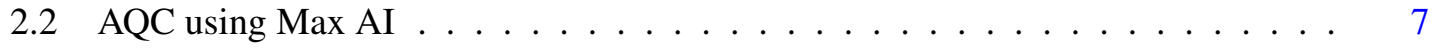

2.3 RCNN overview . . . . . . . . . . . . . . . . . . . . . 13

2.4 Fast RCNN overview . . . . . . . . . . . . . . . . . . . . . . . . 15

2.5 Faster RCNN overview . . . . . . . . . . . . . . . . . . . . . . . 18

2.6 YOLO Model . . . . . . . . . . . . . . . . . . . . . . . . 20

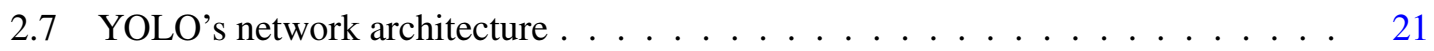

2.8 RetinaNet overview . . . . . . . . . . . . . . . . . . . 23

3.1 Illustrative images and respective annotations. . . . . . . . . . . . . . . . 27

4.1 Cascaded Region Proposal and Classification . . . . . . . . . . . . . . . . 32

4.2 CNN Architecture 1. . . . . . . . . . . . . . . . . . . 33

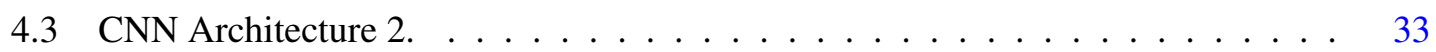

4.4 CNN Architecture $3 . \ldots \ldots \ldots . \ldots \ldots$

4.5 Material Based Hierarchical Approach . . . . . . . . . . . . . . . . . 35

4.6 Shape Based Hierarchical Approach . . . . . . . . . . . . . . . . . 36

A.1 Hierarchical shape based method - detection examples $\ldots \ldots \ldots$ 


\section{List of Tables}

3.1 Frequency of each class in the dataset. . . . . . . . . . . . . . . 28

5.1 Flat method - mAP score . . . . . . . . . . . . . . . . . . . . 39

5.2 Optimizer's hyperparameters . . . . . . . . . . . . . . . . . . . . 40

5.3 Architecture/Optimizer Performance . . . . . . . . . . . . . . . . . . . . 40

5.4 Architecture/Learning Rate Performance . . . . . . . . . . . . . . . . . . . 40

5.5 Hierarchichal Method - Material Based - CNN training results . . . . . . . . . . 42

5.6 Hierarchichal Method - Material Based - CNN training results . . . . . . . . . . 43

5.7 Cross-Sensor Validation mAP score . . . . . . . . . . . . . . . . . . . . 43

5.8 Model Results . . . . . . . . . . . . . . . . . . . . . . . . . . . . . 44

5.9 Label Semantic mAP score . . . . . . . . . . . . . . . . . . . . . . . . . . 45

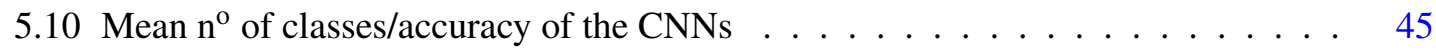

5.11 Individual AP - Part I . . . . . . . . . . . . . . . . . . 46

5.12 Individual AP - Part II . . . . . . . . . . . . . . . . . . . . 46

5.13 Individual AP Set 1 - Part I . . . . . . . . . . . . . . . . . . . 46

5.14 Individual AP Set 1 - Part II . . . . . . . . . . . . . . . . . . . 46

5.15 Individual AP Set 2 - Part I . . . . . . . . . . . . . . . . . . 47

5.16 Individual AP Set 2 - Part II . . . . . . . . . . . . . . . . . 47 


\title{
Abbreviations
}

\author{
aLDA augmented Latent Dirichlet Allocation \\ AP Average precision \\ AQC Autonomous Quality Control \\ CE Cross Entropy \\ CNN Convolutional Neural Network \\ FP False Positive \\ FPN Feature Pyramid Network \\ FN False Negative \\ GLCM Gray Level Co-occurrence Matrix \\ GPRS General Packet Radio System \\ IoU Intersection over Union \\ KNN K-Nearest Neighbor \\ LWW Labeled Waste in the Wild \\ mAP Mean Average Precision \\ MLP Multilayer Perceptron \\ MNIST Mixed National Institute of Standards and Technology \\ ReLU Rectified Linear Function \\ RFID Radio Frequency Identification \\ RGB Red-Green-Blue \\ RPN Region Proposal Network \\ RoI Region of Interest \\ SGD Stochastic Gradient Descent \\ SIFT Scale Invariant Feature Transform \\ SN Sensor Network \\ SVD Singular Value Decomposition \\ SVM Support Vector Machines \\ TP True Postive \\ VIS Visual Identification System \\ WVC Workshop de Visão Computacional \\ YOLO You Only Look Once
}





\section{Chapter 1}

\section{Introduction}

Machine vision is essential to any modern automated system, it is used to provide imaging-based automatic inspection and analysis. This dissertation is focused on the software branch of the automation of waste recycling, and focused on the vision branch of the software. The goal is to develop a deep learning technology to identify different objects and materials on used food trays. Deep Learning is being successfully applied in diverse areas such as autonomous driving, medical imaging and multiple industrial environments. Using an intelligent object identification software in waste recycling is an advantageous approach when compared to the traditional sorting methods. This is because the traditional approach is based on human good will and labour, which are prone to fail. Also, deep learning techniques are able to identify a larger quantity of different objects in a shorter period of time.

\subsection{Motivation}

"Action on climate change is urgent. The more we delay, the more we will pay in lives and in money" said Ban Ki-Moon, former Secretary-General of the United Nations [2]. We must bear in mind that global warming does not only affect human kind but also wildlife, on a very serious level.

A more efficient waste sorting will increase recycling. This will translate in less polluted oceans and reduced harvesting of raw material, preserving wildlife. Portugal failed to meet the waste policy proposed by the European Commission [3], and the United Nations wants to ensure environmental sustainability by 2030 [4]. It is only possible to achieve such targets through an appropriate application of today's technology.

On our daily lives we may forget to separate correctly the waste of our homes, and industrially speaking the companies responsible for this part have to spend a lot on labor and heavy machinery. As deep learning is growing in the field of object detection, and off-the-shelf hardware is computationally capable of processing those algorithms, it seems promising to apply deep learning methods to waste separation, increasing the quantity of recycled material. This translates in an easier day-to-day life for the common person and more efficacy for the industry. 
The main goals of this dissertation are: Firstly, to identify the different objects within the food tray, followed by recognizing what is waste and what is not. Finally, within the identified waste, the aim is to sort its different types.

Some difficulties expected to be tackled are: issues with pieces of waste covering other pieces (e.g. napkin covering an empty cup of plastic); transparent garbage (e.g. empty bottles of water, glass); and the dataset available, which might be limited.

In this work some of the previous identified limitations are addressed, making two main contributions: a) the proposal of a hierarchical deep model for waste detection and classification; b) a novel dataset is also presented and will be made freely available to the research community for benchmark purposes. This dataset has various possible applications, such as: waste detection and classification, which types of waste are produced by different restaurants, or even to figure out which piece of garbage is the most common. To our knowledge it will be the first dataset in this field.

The solution aims to reduce the dependency on human sorting in households and industrial sorting plants. This means that the challenge is the accuracy of the algorithm, the real-time detection and its readiness to be applied on different robots for different applications.

Climate change does not respect boarders, it's a global problem demanding a global solution.

\subsection{Objectives}

The project has a well set of detailed objectives, verifiable, challenging but achievable, seen in the list bellow:

- Develop a new dataset composed by used food trays;

- Study the performance of state-of-the-art algorithms in the developed dataset;

- Develop a scalable deep learning model to apply on our dataset;

- Comparative analysis of the results of the developed model with the state-of-the-art solutions;

- Propose a Real-World ready solution.

In the case of accomplished objectives, the scientific community has a novel dataset, with uncontrolled real-world images, meaning that algorithms trained on such dataset are closer to a real-world application. A study on the performance of the state-of-the-art algorithms for object detection and classification applied to waste sorting enables further discussion on the matter. If a reasonable solution is presented, than more projects can start on the bases of that solution, such as a robotic application or a solution for video based system for industrial application. At the moment, a scientific paper, submitted to the WVC 2019 conference, is waiting for review. The dataset and proposed solution, are described and analyzed in the submitted paper. 


\subsection{Dissertation Structure}

The dissertation first considers, in addition to the introductory chapter, the state-of-the-art in waste sorting in chapter 2. It then provides some background knowledge of deep learning techniques and the state-of-the-art algorithms in object recognition. Next, chapter 3 presents the novel dataset, including the explanation of some relevant characteristics. The methodology that led to the solutions, and the solutions themselves are described in chapter 4 . Chapter 5, includes the experimental results, featuring the tests and metrics used to calculate the performance of the developed models. Lastly, conclusions are drawn in chapter 6 with a few suggestions for future research. 


\section{Chapter 2}

\section{State Of The Art}

The present chapter is divided in 5 sections. In section 2.1, are analyzed the traditional sorting techniques, used in industrial recycling plants. Some relevant commercial solutions, that are based in artificial intelligence models, are presented in section 2.2. Related work regarding intelligent waste sorting is discussed in section 2.3. Section 2.4 includes background knowledge on deep learning techniques, emphasizing on convolutional neural networks. Followed by section 2.5, which considers the state-of-the-art architectures in object recognition.

\subsection{Sorting Techniques}

Conventional waste disposal factories will typically use one or more of the following methods:

- Trommel separators: Sorts materials according to their particle size.

- Eddy current separator: This method is specifically for the separation of metals. A current occurs when a conductor is exposed to a changing magnetic field dividing ferrous from non ferrous metals.

- Induction sorting: Sensors locate different types of metal which are then separated by a system of fast air jets which are linked to the sensors.

- Near infrared sensors: The NIR sensor distinguishes between different materials based on the way they reflect light.

- X-ray: Used to distinguish between different types of waste based on their density.

- Manual Sorting: Manual sorting of waste is still very much a technique that is used in the world today. Material is sorted by hand on manual picking lines. 
Most waste sorting plants are equipped with these techniques, but most of them alone are not capable of reaching a recyclable ready sorting. For instance the trommel separator sorts by size, which may be helpful for large quantities of waste, making sure small pieces are not lost in the process. The issue is it does not sort by material.

Eddy current and Induction sorting are techniques to sort metals only. However there is a problem, the air jets may be imprecise, catching other pieces of waste within range and wrongly segregate.

The NIR sensor and X-ray techniques distinguish between different types of waste but usually with better results on pre-sorted waste [5], moreover, milk cartons for instance, do reflect light as a cardboard container but its inside is made of plastic, causing an error of separation, this applies to mixed packaging.

A technique regularly present is manual sorting, as shown in Figure. 2.1, it is by definition preformed by humans and it is prone to human error, although it is great to sort outliers (ie. rare types of waste or in case of machine failure). However the overall performance is poor mainly because the ability to make mistakes is increased after long hour shifts of mechanically repeated movements and it is possible to see that in the recycling rates published by the European Environment Agency [6].

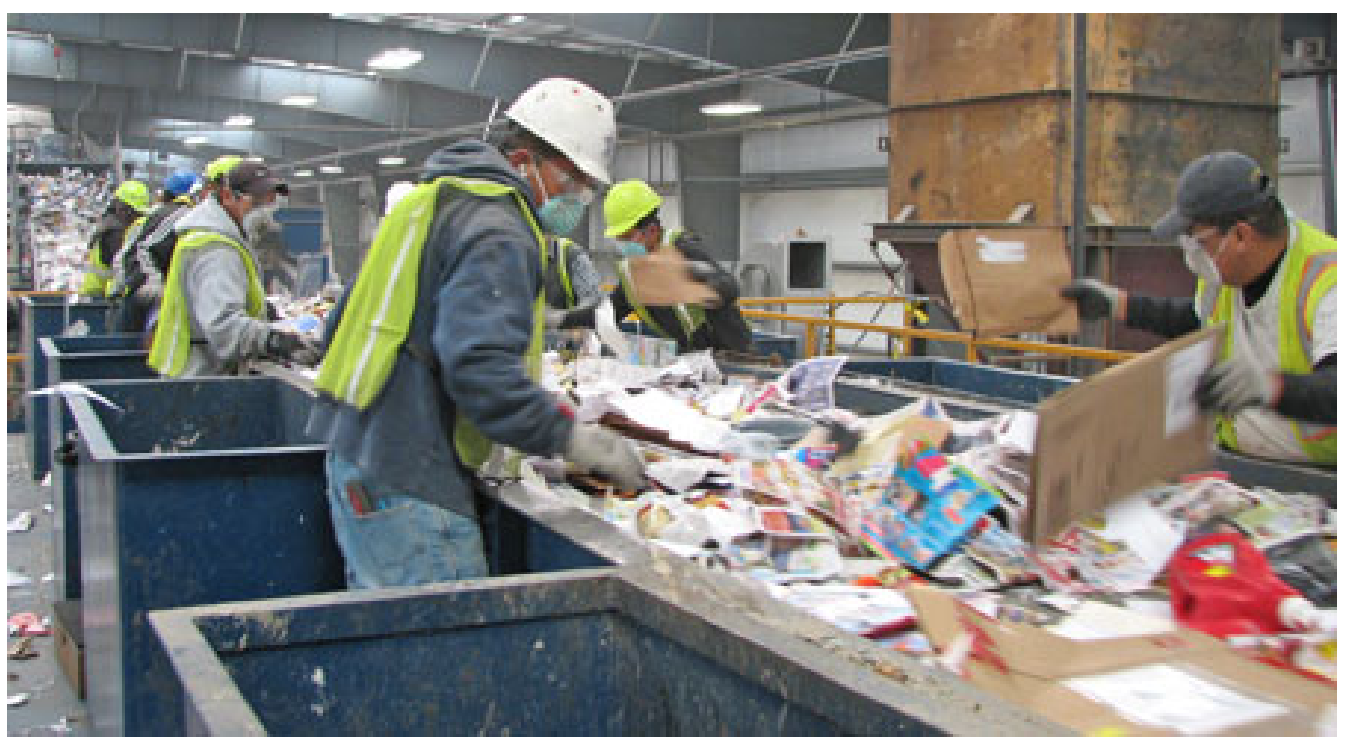

Figure 2.1: Sorting waste by hand [1] 


\subsection{Commercial Solutions}

Bulk Handling Systems has announced Max-AI [7], a deep learning technology that identifies recyclables, employing multi-layer neural networks and a vision system. There are two solutions that use this technology:

- Autonomous Quality Control: The AQC, seen in Figure. 2.2, was the first product to make use of Max-AI, its concept is to see what is on the belt and make decisions, it tells a robotic sorter what to do. Pick "item X" and place into "chute 1" then pick "item Y" and placing into "chute 2.". It sorts items at speeds of up to 65 picks per minute, the AQC compliments Max-AI's optical sorters to create a completely autonomous product sorting of containers and paper.

- Visual Identification System: VIS uses the same technology as the AQC, but without the robotic sorting. Max-AI is at work in VIS to identify materials, capture and communicate these data for other uses. It can be used to verify the quality of end products, or to analyze residue as it leaves a system.

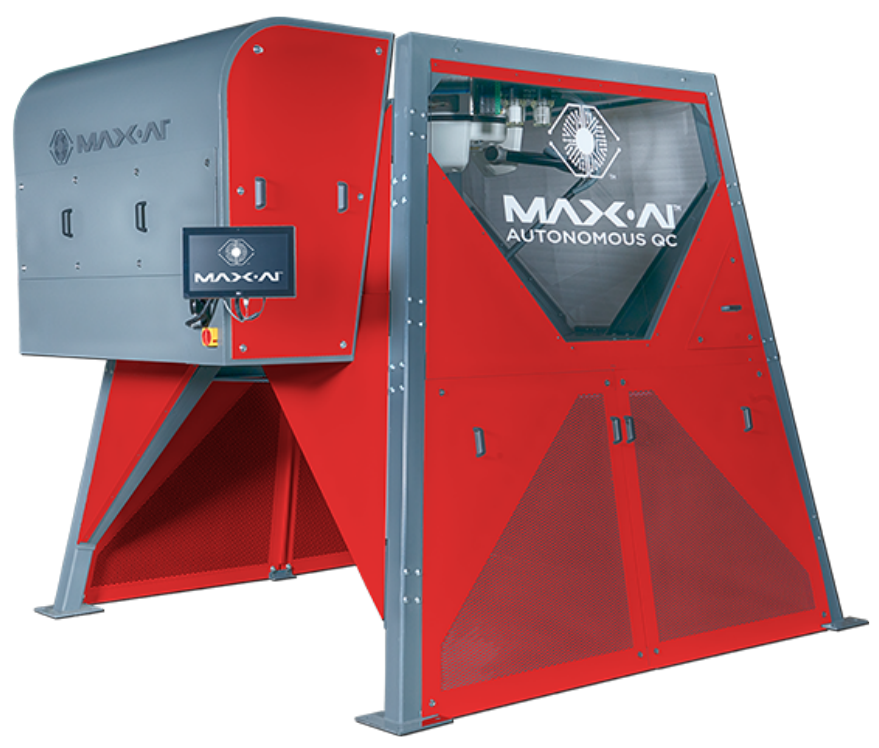

Figure 2.2: AQC using Max AI

Even thought there is not much information about these solutions, they are prone to the systematic problems caused by the technologies they claim to use. For instance, the garbage must be spread through the conveyor belt, several problems surge if these restriction is not verified, the algorithm may not recognize a type of waste if the one is covered by another, the robot that picks the trash might not be fast enough to pick all of the trash. Pick and place robots usually have a maximum payload, this means that the waste that is being sorted may weight more than that maximum that the robot can pick. 


\subsection{Related Work}

Most solutions are image-based, different techniques have been developed and used in waste classification as discussed in [8].

Classification Based on Shape Sergie et al [9] developed a technique to measure similarities between shapes and uses the result to classify the object. The similarity of objects is measured by detecting the co-occurrence between points chosen on the shapes considered, and by using the conformity to use an aligning transform. The symmetry issue is solved by assigning a descriptor as the shape context to each chosen point, and the remaining points are retrieved relatively to the shape context at the reference point. Corresponding points on two similar shapes have identical dimensional contexts, the transformation that best coordinates the two shapes is calculated. The sum of diffeences between the two corresponding points, with a terminology that calculates the weight of the aligning transform, is used to estimate the deviation between the shapes. Deciding if the shape is similar to the one in the image is done by a nearest-neighbour classification problem. This method obtains accurate descriptors for point sets, shape recognition and dimensionality matching.

Classification Based on Reflectance Properties A method that classifies reflectance properties of surfaces under uncontrolled real-world illumination was developed in [10]. The reflectance estimation algorithm learns correlations between surface reflectance and the data enumerated on an observed image with surfaces with random geometry. This paper introduces a technique for classifying using the reflective property of objects, from monochromatic images.

Classification Based on Materials This method was developed in Ce Liu et al [11], and it is based on material recognition. It is a difficult task to find reliable features that can differentiate materials into different classes compared to the popular methods that are used for object recognition. The authors used a set of low and mid-level features that are used to learn and obtain various aspects of material appearance. An augmented Latent Dirichlet Allocation (aLDA) model is developed, in order to combine multiple features under a Bayesian framework, creating an ideal combination of features. This implementation of a model that recognizes the objects depending on their materialistic properties is pioneer in the scientific community.

Classification using Convolutional Neural Networks AlexNet [12] is definitely one of the most popular CNN models used for image classification. Since its development, different CNNs have been used on object recognition and the results obtained show that a large and deep CNN can output very good results on any complicated dataset using supervised learning methods. Being the method with better results, and tha basis of the solution, a detailed overview is in section 2.4. 


\subsubsection{State-of-the-Art solutions in waste classification}

Over the years, different studies have been implemented with the aim of minimizing the impact of the uncontrolled disposal of waste. Technologies such as Radio Frequency Identification (RFID) and Sensor Network (SN) have been used to provide a new way to optimize waste management systems such as in [13], where the authors proposed a method to improve the quality of selective collection by tracking the waste stream of a city. Each pile of waste is detected from information stored on an RFID tag and during the waste processing phase, RFID tags are read to provide important information. Similar methods are used in [14] to estimate household waste, in [15] it is discussed different RFID applications on management of solid waste on a municipal level. Nevertheless, neither of these studies assist in a direct waste sorting method.

Maher Arebey et al. [16] propose the gray level co-occurrence matrix (GLCM) method for garbage detection and classification, combining advanced communication mechanisations with GLCM to strengthen the waste assembling operation. The proposed system uses several communication technologies including radio frequency identification (RFID), geographical information system (GIS) and general packet radio system (GPRS) integrated with a camera, streamlining the solid waste monitoring and management. The features are obtained from the GLCM and then used as inputs to a multilayer perceptron (MLP) and a K-nearest neighbor (KNN) methods for garbage separation. The results obtained show that the KNN classifier surpasses the MLP.

Sakr et al. [17] tries to automatize waste sorting by applying machine learning algorithms to classify waste in images. The authors use two popular methods, deep learning with convolutional neural networks (CNN) [18] and support vector machines (SVM) [19].The methods used create a different classifier that sorts waste into 3 main classes (plastic, paper and metal) using $256 * 256$ coloured images of the waste. The accuracy produced by the models of the two classifiers are compared in order to choose the best one. The adopted model was implemented on a raspberry pi 3, that controlled a mechanical system that physically sorted the waste, placing it into the corresponding container. The results obtained show that SVM achieved a classification accuracy of $94.8 \%$, while the CNN achieved an accuracy of $83 \%$. The training images were scaled down from the original $256 * 256$ size to $32 * 32$ size. This reduction resulted in overfitting problems. The model produced in this research had an average execution time of 0.1 seconds on a raspberry pi 3.

AutoTrash [20] was created at TechCrunch Disrupt Hackathon. An automatic sorting trashcan that sort waste within two classes, recyclable and compost. Their model has a rotating top where a raspberry pi camera is placed. The team used google's tensorflow AI engine and built their own layer on top of it for object recognition. The trashcan is divided into two different containers, and the rotating top of the trashcan places the object on their respective containers based on their classification.

In Mindy Yang et al. [21], the authors created a model that classifies waste into six different classes. The dataset used was hand collected and consisted of at least 400 images for each of the classes considered. Support Vector Machines with scale invariant feature transform (SIFT) [22] 
features, and Convolutional Neural Networks were used to classify the images. Torch7 framework for Lua was used to construct the CNN classifier. An eleven-layer CNN architecture was developed. Experiments show that the SVM performs more efficiently than the CNN. The accuracy obtained was $63 \%$, the training error was $30 \%$. The CNN used was not trained to its full potential.

In a similar work, Oluwasanya Awe et al. [23] proposed a method using Faster Region-based Convolutional Neural, Networks (Faster R-CNN) [24], technique to get region proposals and object classification, reaching a mAP score of $68.3 \%$. The waste was categorized into three classes (landfill, recycling, and paper).

The authors of [25] propose an automated system based on a deep learning approach and traditional techniques to correctly separate waste into four different trash classes (glass, metal, paper and, plastic). Results showed that VGG-16 [26] methods are an efficient approach for this problem, reaching $93 \%$ of accuracy on its best scenario.

The authors of RecycleNet [27] experimented on widely recognized deep convolutional neural network architectures. Training without pre-trained weights, Inception-v4 surpassed all others with 90\% test accuracy.The authors then applied transfer learning and fine-tuning of weight parameters using ImageNet weights, and DenseNet121 obtained the best result with 95\% test accuracy.This latter solution has slower prediction time.

In the last years, computer vision has been considered as a tool to support waste sorting, and deep learning techniques have reached reasonable results in controlled scenarios. Object detection, less addressed in waste management, was considered in Oluwasanya Awe et al [23] using the Faster R-CNN model, showing reasonable results.

\subsection{Background Knowledge}

The field of computer vision has been evolving rapidly due to deep learning techniques, and currently those techniques are the state-of-the-art solutions in many object detection and classification problems. As mentioned before the goal is to develop a deep learning technology to identify different objects and materials on used food trays. Deep learning in this context will be composed by machine learning methods and image processing. [28] Machine leaning methods try to discover a model by using a set of input data and change the algorithm, those changes are influenced by the inputs in order to learn [29]. There are several solutions in the deep learning field, the one that is going to be studied is Convolutional Neural Network (CNN) and its variants. [18]

\subsubsection{Convolutional Neural Network}

Convolutional Neural Networks are the most well known deep learning model used to solve image classification tasks. Recently, CNN features are also used in object recognition models to predict object bounding boxes. CNNs can be decomposed in basic building blocks such as convolutions, pooling operators, activation functions, and fully-connected layers. 
- Convolutional Layer: Is composed by a set of image filters, each to be applied to several regions of the input, called local receptive field. The filter is a $k \times k$ matrix of weights, and each weight is a parameter of the model to be learned. The output is a linear combination of pixel values defined by the size of the filter.

- Activation Function: In this block the rectified linear function (ReLU) is often used after convolution or fully connected layers, it cancels all negative values and it's linear for all positive ones. This is related to the non-negativity constraint used in image processing to regularize methods based on subspace projections. The main advantage for this, is that it might cancel some neurons that detect objects that are not present in the image. It is possible to allow small negative values to be used in training and the activation function is called Leaky ReLU.

- Feature Map: A vector produced by a convolution neuron passes through an activation function is called a feature map, those maps form a tensor that is then used as an input for the next layer.

- Pooling: Used to downsample the image in order to reduce the spatial dimension of the vector, usually the maxpooling operator is the choice. It reduces the size of data and also the size of the image obtaining a multi-resolution filter bank. There are some proposals to discard the pooling block and replace it with a larger stride in the convolution layers.

- Normalization: Normalization is applied to the input and to the output of convolutional layers. For input layers it is common to apply the z-score normalization that can be described as a whitening process and for the following layers usually the channel-wise normalization is applied, using L1-norm or L2-norm. AlexNet uses Local Response Normalization, GoogLeNet and ResNet use Batch Normalization. Batch Normalization has been used to reduce overfitting in $\mathrm{CNN}$ training.

- Fully Connected Layer: Often included after many convolution layers in order to learn weights to classify the representation. It takes the full input vector with the data from all the layers, and produces a scalar value. Usually the last layer of a CNN outputs the class membership for each class, commonly using softmax or support vector machines.

- Loss Function: A loss function is used to measure the performance of the model given the input and expected output, the output of this function is vital in the training of the network, it will affect the update of the weights and help in the optimization.

- Optimization: A defined loss function enables the network to update the parameters of the CNN to minimize the loss function's value. For that there are several optimization algorithms, in CNN is common to use Stochastic Gradient Descent (SGD), Momentum, AdaGrad, RMSProp and Adam. 


\subsubsection{Frequently Used CNNs}

With the success of CNN applications, some architectures became extremely popular after achieving great accuracy in image classification, and when used as subroutines in other algorithms to solve different tasks. Some of the commonly used architectures are AlexNet, ResNet and GoogLeNet. A brief overview of these CNNs is seen below.

- AlexNet [12]: Was originally designed in two branches for parallel processing. It uses Local Response normalization, maxpooling with overlapping, a batch size of 128 examples, momentum of 0.9 and a decay of 0.0005 . Weights were initialized with a gaussian-distributed random values, with a standard deviation of 0.01 , and a bias of 1 for the second, forth and fifth convolutional layers. And bias 0 for the remaining layers. A learning rate of 0.01 and randomly dividing it by 10 three times during training. AlexNet had the best performance at the ImageNet Challenge of 2012 [30].

- VGG-Net [26]: Was designed to increase depth with filters of size of at most $3 \times 3$. It is said that stacking three covolutional layers with $3 \times 3$ filters has an advantage of incorporating more rectification layers making the decision function more discriminative when compared with a single $7 \times 7$ filter layer. Two most known versions are VGG-16, with sixteen weight layers and VGG-19 with nineteen weight layers. During training, the batch size used is 256 . Maxpooling window size of $2 \times 2$, and stride 2. It is regularized by weight decay using L2 regularization of 0.0005 and dropout of 0.5 . For the first two fully connected layers all bias are set to 0 and the learning rate behaves similarly to the one used in AlexNet.

- ResNet [31]: When the number of layers is increased the accuracy first saturates and then starts to degrade and it becomes unfit for training. The authors of Resnet proposed a method for networks with 34 to 152 layers. The authors claim that it is an optimization problem, and they designed blocks that preserve the characteristics of the original vector, before its transformation in a layer and sum it to the output of the layer. Because the gradient is an additive term, it is not probable to vanish even with many layers. This architecture does not use fully connected layers. After the last layer the output is obtained by an average pooling.

- GoogLeNet [32]: It is based on modules called Inception, later improvements such as Inception V3 include decrease of representation size from input to output, use of higher dimensional representations per layer, with lower dimensional embedding with $1 \times 1$ convolutions before spatial convolutions, and balance between the number of filters and number of layers. The Inception module explores the same idea of VGG-Net of break larger filters into smaller consecutive filters, but Inception stacks different sequences, in parallel, of small convolutions and concatenates the outputs of the different parallel sequences. The authors used a label-smoothing regularization (LSR) that constrains the last layer to output a non sparse solution. For training was also used gradient clipping with threshold 2, and all other parameters were standard. In the ImageNet Challenge of 2014 [30], GoogLeNet and VGG achieved similar results. 


\subsection{State-of-the-Art in visual object detection algorithms}

This section introduces the state-of-the-art algorithms in context to our problem, each method provides vital knowledge regarding object detection and classification as well as network training for scarce datasets and uncontrolled backgrounds.

\subsubsection{R-CNN}

The algorithm is a combination of region proposals and CNN features, hence the name R-CNN [ [33] [34]]. This approach combines two key insights, it applies high capacity CNNs to bottomup region proposals to localize and segment objects, and when labeled training data is scarce, supervised pre-training for an auxiliary task, followed by a domain-specific fine-tuning proved to be a significant performance boost. The system takes an image as an input, extracts around 2000 bottom-up region proposals, computes features using CNNs and then classifies each region using class-specific SVMs. In Figure. 2.3 an overview of the R-CNN architecture is depicted.

\section{R-CNN: Regions with CNN features}
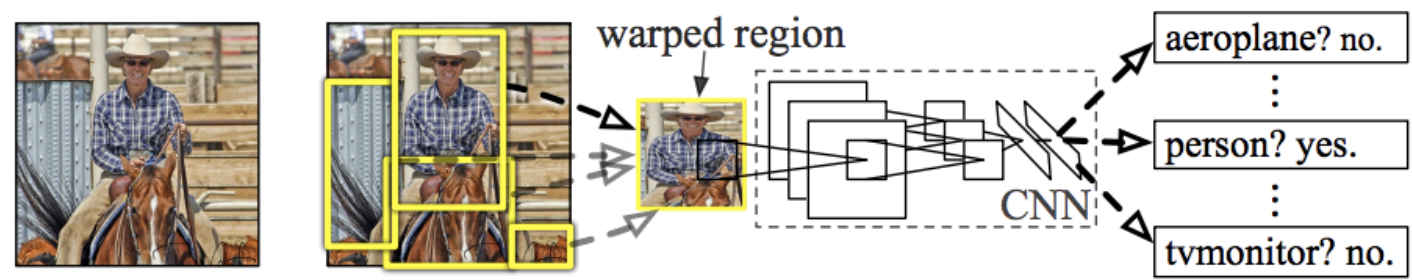

1. Input image
2. Extract region proposals $(\sim 2 \mathrm{k})$
3. Compute $\mathrm{CNN}$ features
4. Classify regions

Figure 2.3: RCNN overview

\subsubsection{Region Proposals}

There are several methods of generating region proposals, in [33] it is said that R-CNN is agnostic to the method, but selective search [35] was chosen. This method takes an image as an input and outputs several bounding boxes corresponding to all patches in the image that are likely to be objects, and are called region proposals. These region proposals can be noisy, overlapping and may not entirely contain the object, but amongst these region proposals some will be very close to the actual object. In R-CNN these region proposals go through the CNN to extract its features and are then classified. In the dataset used, an average of 2403 regions per image were generated. 


\subsubsection{Network Architectures}

The authors found out that the choice of the network has a large effect on the performance, for most results the network used was AlexNet. They compared this network with the 16 layer deep network proposed by Simonyan and Zisserman and refer to it as "O-Net", this network was one of the top performers in the ILSVRC 2014 challenge, it has a homogeneous structure consisting of thirteen layers of $3 \times 3$ convolutional kernels, with five max pooling layers interspersed, and topped with three fully connected layers. When compared, O-Net outperformed AlexNet with a mean average precision (mAP) score of $66.0 \%$ but the computing time of the forward pass was also seven times longer than AlexNet.

\subsubsection{Bounding Box Regression}

Based on error analysis the authors implemented a simple method to reduce localization errors. They train a linear regression model to predict a new detection window given the pool features for selective search region proposal. After scoring each region proposal with a class specific SVM, a new bounding box is predicted using a bounding-box regressor, this regressor uses the features computed by the CNN. Given a set of pairs $P$ and $G$, where $P$ specifies the pixel coordinates of the center, height and width of the proposal bounding box, and $G$ specifies the ground-truth bounding box in the same way. A set of transformations to predict a ground-truth bounding box are applied. The transformation is modeled as a linear function of the pool features of the proposal $P$, thus from the dependence of $P$, a learnable parameters vector is obtained and trained, using regularized least squares objective. This method proved to fix a large number of mislocalized detections, boosting the mAP score by at least $3 \%$.

\subsubsection{Training}

The training approach was carefully defined due to the lack of labeled bounding boxes. The authors divided the training in two different phases as described below.

- Supervised pre-training: The $\mathrm{CNN}$ was discriminatively pre-trained on a large dataset using image-level annotations only, it was preformed using the open source Caffe CNN library. The CNN used, nearly matched the results of AlexNet, obtaining a top-1 error rate $2.2 \%$ higher due to simplifications in training.

- Domain-specific fine-tuning: To adapt the CNN used to the specifications of the task, the authors continued training using stochastic gradient descent(SGD) with warped region proposal only. The $\mathrm{CNN}$ was initialized with random $(N+1)$-way classification layer, where $N$ is the number of classes plus one for background. For the VOC dataset $N=20$ and for the ILSVRC2013 $N=200$. For all region proposal, the IoU overlap was bigger than 0.5 with a ground-truth box as positives for that box's class and the rest as negatives. SGD was started at a learning rate of 0.001 which allows fine-tuning to make progress while not clobbering the initialization. In each SGD iteration it was uniformly sampled 32 positive 
windows and 96 background windows to construct a mini-batch of size 128. The sample was bias towards positive windows due to their rarity.

Object category classifiers. Once features are extracted and training labels are applied, one linear SVM per class is optimized. Since the training data was too large to fit in memory, the authors applied a method that allows hard negative mining to converge quickly, empirical testing shows that that mAP score stops increasing after just one pass.

\subsubsection{Summary}

Before R-CNN object detection performance had stagnated. R-CNN represents a simple and scalable object detection algorithm that gives 30\% relative improvement on PASCAL VOC 2012 [36]. There is room from improvement in the R-CNN algorithm and that is discussed further. The training techniques applied to small datasets will be important to the thesis' development.

\subsubsection{Fast R-CNN}

The same author of [33] solved some problems of that algorithm and called it Fast R-CNN [37]. Current object detection algorithms, due to their complexity, train models in multi-stage pipelines that are slow. Detection creates two primary challenges, the first one being that numerous amounts of region proposals must be processed, and the second being that these proposals usually don't contain the entirety of the object and must be refined. As seen in Figure. 2.4, Fast R-CNN proposes a single-stage training algorithm that jointly learns to classify object proposals and refine their spatial location.

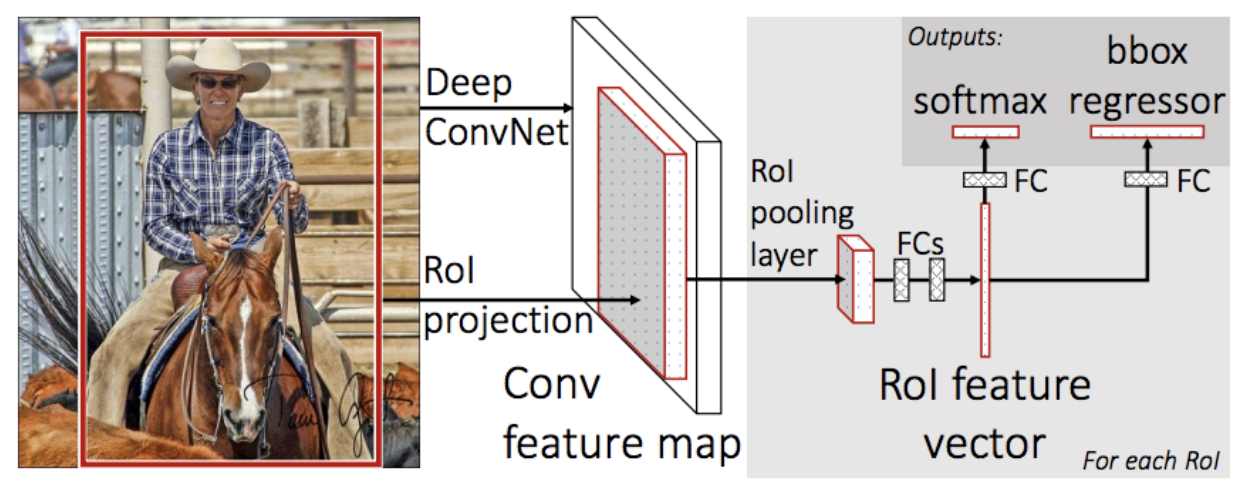

Figure 2.4: Fast RCNN overview

\subsubsection{Architecture}

This network takes as input an entire image and a set of object proposals. The network first processes the image with several convolutional and max pooling layers to get a convolutional feature 
map. Then, for each object proposal a region of interest pooling layer extracts a fixed-length feature vector from the feature map. Each feature vector is fed into a sequence of fully connected layers that branch into two sibling output layers. The first produces a softmax probability estimates over $N$ object classes plus a background class. The second, outputs four real-valued numbers for each of the $N$ object classes. Each set of four values encodes refined bounding-box coordinates for one of the $N$ classes. Region of interest max pooling works by dividing the window in subwindows and then max-pooling the values in each sub-window to the corresponding output grid cell.

For image classification, the time spent computing the fully connected layers is small when compared to the convolutional layers, opposed, for detection, the amount of regions of interest to process is large, and half of the forward pass time is spent computing the fully connected layers. These are easily accelerated by compressing them, using the truncated SVD (Singular Value Decomposition).

\subsubsection{Training}

The authors used three different networks, each with five max pooling layers, and between five and thirteen convolutional layers, then when a network initializes a Fast R-CNN network, it undergoes three transformations. First, the last max pooling layer is replaced by a region of interest pooling layer, that is configured by setting compatible height and width with the network's first fully connected layer; Second, the network's last fully connected layer and the softmax classifier are replaced with the two sibling layers described in the architecture; Third, the network is modified to take a list of images and a list of regions of interest in those images.

Training all network's weights with back-propagation is a characteristic of Fast R-CNN. A method that takes advantage of feature sharing during training is proposed. Stochastic gradient descent mini-batches are sampled hierarchically, regions of interest from the same image share computation and memory in the forward and backward passes. In addition to hierarchical sampling, Fast R-CNN uses a streamlined training process with one fine-tuning stage that optimizes a softmax classifier and bounding-box regressors. This procedure's components are the following:

- Multi-task Loss: The second sibling layer of Fast R-CNN outputs bounding-box regression offsets $t k$, for each of the $K$ object classes, it is used the parameterization for $t k$, in which $t k$ specifies a scale-invariant translation and log-space height/width shift relative to an object proposal. Each training region of interest is labeled with a ground-truth class $u$ and a groundtruth bounding-box regression target $v$. A multi-task loss $L$ on each labeled region of interest is used to jointly train for classification and bounding-box regression.

- Mini-batch Sampling: Each stochastic gradient descend mini-batch is constructed from 2 images, chosen at random. The mini-batches used are of size 128, sampling 64 regions of interest (RoIs) from each image. It is taken $25 \%$ of the RoIs from object proposals that have intersection over union (IoU) overlap with a ground- truth bounding box of at least 0.5. These RoIs comprise the examples labeled with a foreground object class, the remaining 
RoIs are sampled from object proposals that have a maximum IoU with ground truth in the interval $[0.1,0.5]$. The lower threshold of 0.1 appears to act as a heuristic for hard example mining. During training, images are horizontally flipped with probability 0.5 . No other data augmentation is used.

- Back-propagation through RoI layers: Back-propagation routes derivatives through the RoI pooling layer. Only one image per mini-batch is used. The RoI pooling layer's backwards function computes partial derivative of the loss function of each input variable by following the argmax switches. For each mini-batch RoI, and for each pooling output unit, the partial derivative is accumulated if the input is the argmax selected for the output unit by max pooling. In back-propagation, the partial derivatives are already computed by the backwards function of the layer on top of the RoI pooling layer.

- Stochastic Gradient Descent Hyper-parameters: The fully connected layers used for softmax classification and bounding-box regression are initialized from zero-mean Gaussian distributions with standard deviations 0.01 and 0.001 . Initialize biases with 0 . All layers use a per-layer learning rate of 1 for weights, 2 for biases, and a global learning rate of 0.001. The authors trained on VOC07 and VOC12 trainval and ran SGD for 30k mini-batch iterations, and then lowered the learning rate to 0.0001 and train for another $10 \mathrm{k}$ iterations. When training on larger datasets, run SGD for more iterations, a momentum of 0.9 and parameter decay of 0.0005 are used.

\subsubsection{Summary}

This algorithm reaches top performance on VOC 2007 [38] (70\% mAP), on VOC 2010 [39] (68.8\% mAP) and on VOC 2012 [36] (68.4\% mAP). Fast training and testing when compared with R-CNN and others, and it was able to improve mAP on deep CNNs with fine-tuning.

\subsubsection{Faster R-CNN}

Both R-CNN and Fast R-CNN use selective search to generate region proposals, in [24] the authors came up with an object detection algorithm that eliminates the selective search and lets the network learn the region proposals. The insight of Faster R-CNN is that region proposals depend on features of the image that were already calculated with the forward pass of the CNN and decided to use those results to obtain region proposals. The new method is depicted in Figure. 2.5.

\subsubsection{Region Proposal Networks}

A Region Proposal Network (RPN) takes an image as input and outputs a set of rectangular object proposals, this process is modeled with a fully convolutional network because the ultimate goal is to share computation with a Fast R-CNN object detection network, and it is assumed that both nets share a common set of convolutional layers. To generate region proposals, a network is slid over the convolutional feature map output by the last shared convolutional layer. This network takes 


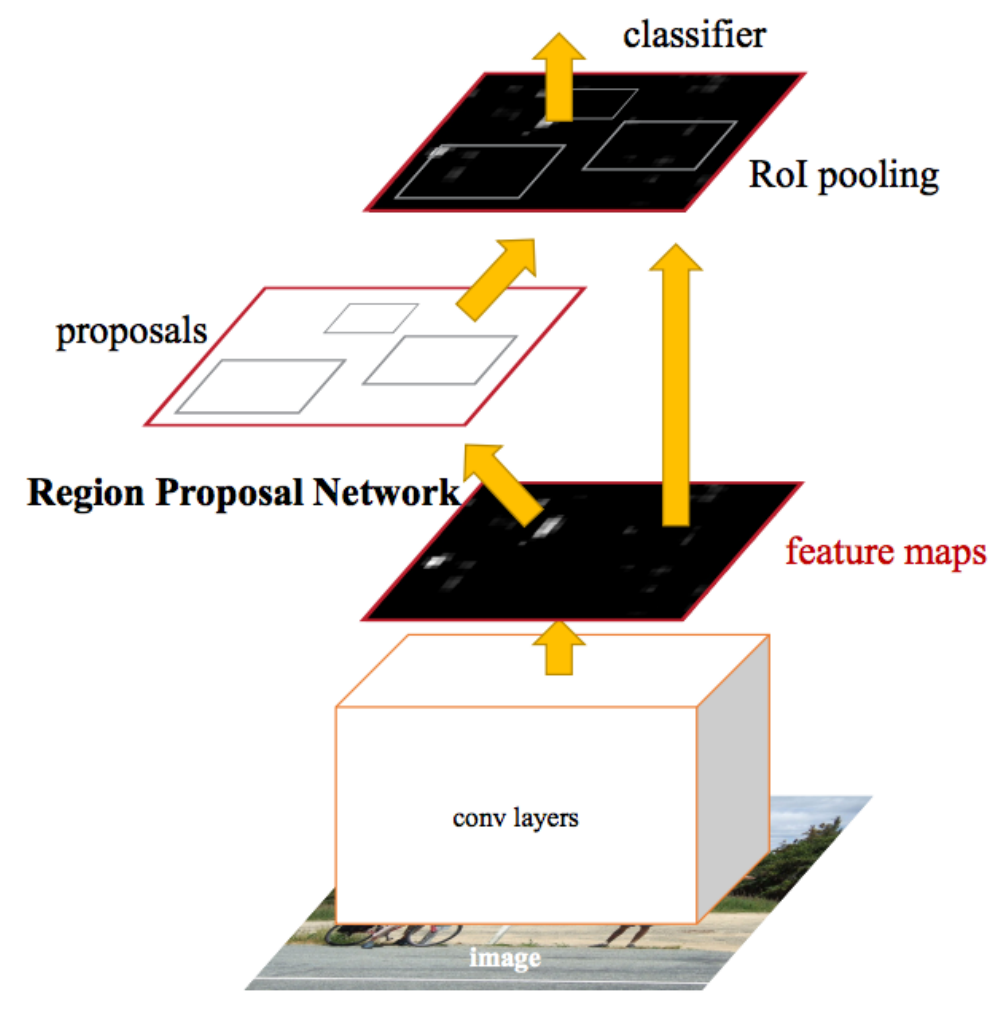

Figure 2.5: Faster RCNN overview

as input a spatial window of the convolutional feature map. Each sliding window is mapped to a lower-dimensional feature. This feature is fed into two sibling fully-connected layers. The first being a box-regression layer, reg, the second being a box-classification layer, $\mathrm{cls}$.

- Anchors: At each sliding-window location, it is simultaneously predicted multiple region proposals, where the number of maximum proposals for each location is denoted as $k$. So the reg layer has $4 k$ outputs encoding the coordinates of $k$ boxes, and the $c l s$ layer outputs $2 k$ scores that estimate the probability of containing an object or not contain an object for each proposal. The $k$ proposals are parameterized relative to $k$ reference boxes, which are called anchors. An anchor is centered at the sliding window in question, and is associated with a scale and aspect ratio. This approach is translation invariant, both in terms of the anchors and the functions that compute proposals relative to the anchors. If an object translates in an image, the proposal and the same function translates and are able to predict the proposal in the new location. It is also multi-scale design based on anchors, that means that it uses the convolutional features computed on a single-scale image. This is a key component for sharing features without extra cost for addressing scales.

- Loss Function: For training RPNs, a binary class label is assigned to each anchor. Then it is assigned a positive label to two kinds of anchors: (1) the anchors with the highest Intersection-over-Union (IoU) overlap with a ground-truth box, or (2) an anchor that has an 
IoU overlap higher than 0.7 with any ground-truth box. Usually the second condition is sufficient to determine the positive samples but it is still adopted the first condition because in some rare cases the second condition may find no positive sample. It is assigned a negative label to a non-positive anchor if its IoU ratio is lower than 0.3 for all ground-truth boxes. Anchors that are neither positive nor negative do not contribute to the training objective. With these restrictions, objective function is developed following the multi-task loss in Fast R-CNN. This method achieves bounding-box regression by using the features used for regression that are of the same spatial size on the feature maps. In case of varying sizes, a set of $k$ bounding-box regressors are learned. Each regressor is responsible for one scale and one aspect ratio, and do not share weights. Like this, it is still possible to predict boxes of various sizes even though the features are of a fixed size, thanks to the design of anchors.

- Training: The RPN is trained end-to-end by back-propagation and stochastic gradient descent. It starts by randomly sample 256 anchors in an image to compute the loss function of a mini-batch, where the sampled positive and negative anchors have a ratio of up to 1 . Also randomly initialize all new layers by drawing weights from a zero-mean Gaussian distribution with standard deviation 0.01 . All other layers are initialized by pre-training a model for ImageNet classification. It is used a learning rate of 0.001 for $60 \mathrm{k}$ mini-batches, and 0.0001 for the next 20k mini-batches on the PASCAL VOC dataset [36], a momentum of 0.9 and a weight decay of 0.0005 and this implementation uses AlexNet.

\subsubsection{Sharing Features}

Both RPN and Fast R-CNN, when trained independently will modify their convolutional layers in different ways. Therefore it is needed to develop a technique that allows for sharing convolutional layers between the two networks. The authors adopt a pragmatic 4-step training algorithm to learn shared features via alternating optimization. In the first step, train the RPN as described before. This network is initialized with an ImageNet pre-trained model and then fine-tuned end-to-end for the region proposal task. In the second step, train a separate detection network by Fast R-CNN using the proposals generated by the step 1 RPN. This detection network is also initialized by the ImageNet-pre-trained model. At this point the two networks do not share convolutional layers yet. In the third step, use the detector network to initialize RPN training, but fixed the shared convolutional layers and only fine-tune the layers unique to RPN. Then the two networks share convolutional layers. After these steps and keeping the shared convolutional layers fixed, fine-tune the unique layers of Fast R-CNN.

\subsubsection{Summary}

This method achieved state-of-the-art mAP score on MS COCO [40], VOC 2007 [38] and VOC 2012 [36] (78.8\%), with much faster training and testing time. In previous methods the time spent by region proposal algorithm was a big part of the test time, with this method, region proposal is nearly cost-free, even though the developers of [41] made it faster but with a lower mAP score. 


\subsubsection{YOLO (You Only Look Once)}

This algorithm reframes object detection as a single regression problem, straight from image pixels to bounding box coordinates and class probabilities. Using this system, you only look once (YOLO) [42], at an image to predict what objects are present and where they are. A single convolutional network simultaneously predicts multiple bounding boxes and class probabilities for those boxes, and YOLO trains on full images and directly optimizes detection performance. A simple model overview is seen in Figure. 2.6.

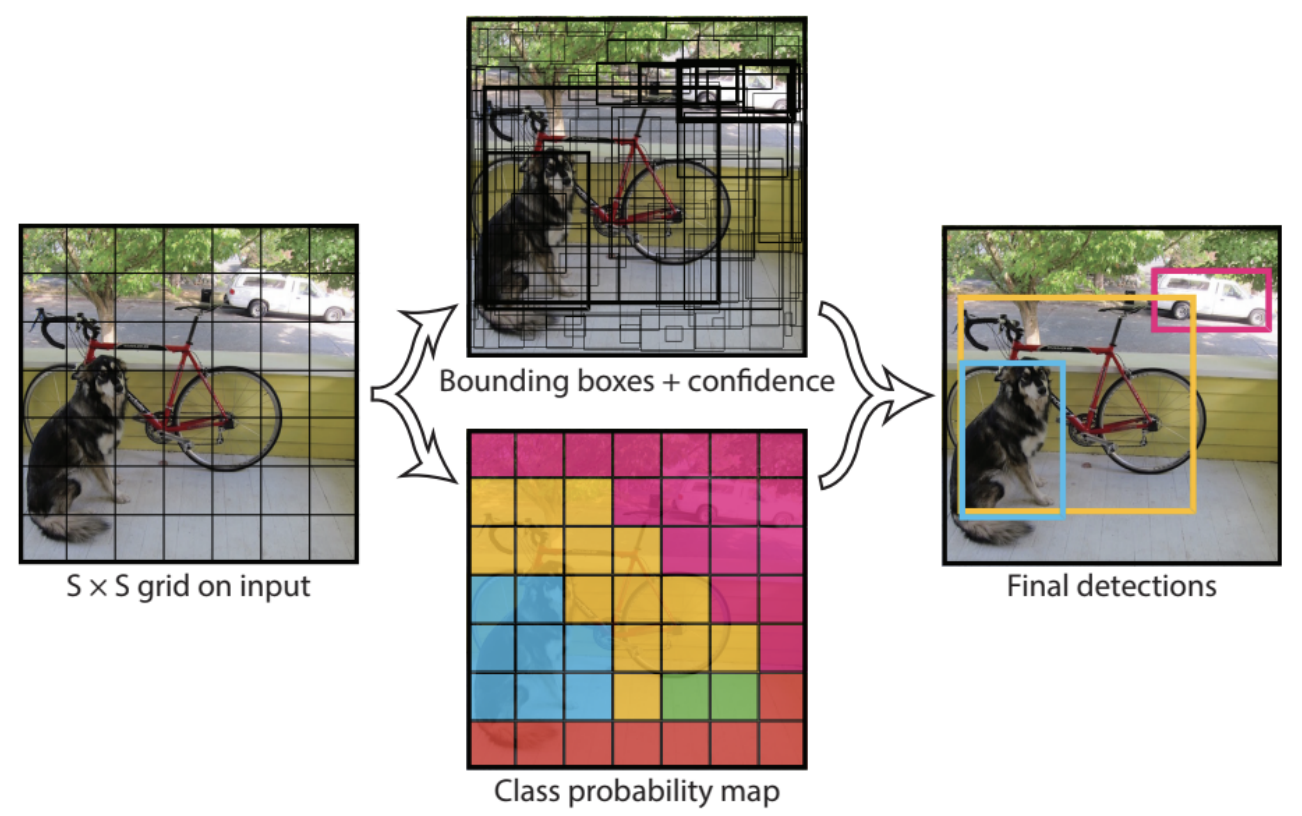

Figure 2.6: YOLO Model

\subsubsection{Unified Detection}

The separate components of object detection are unified into a single neural network and uses features from the entire image to predict each bounding box, it predicts all bounding boxes for all classes simultaneously. The YOLO design enables end-to-end training and real-time speeds while maintaining high average precision.

This system divides the input image into an $S \times S$ grid, if the center of an object falls into a grid cell, that cell is responsible for detecting that object. Each grid cell predicts $B$ bounding boxes and confidence scores for those boxes. If no object exists in that cell, the confidence scores should be zero. Otherwise the confidence score equals the intersection over union (IoU) between the predicted box and the ground truth. Each grid cell also predicts $C$ conditional class probabilities, these probabilities are conditioned on the grid cell containing an object. At test time, the conditional class probabilities and the individual box confidence predictions are multiplied, which 
gives class-specific confidence scores for each box. These scores encode both the probability of that class appearing in the box, and how well the predicted box fits the object.

\subsubsection{Network Design}

This model as a convolutional neural network, the initial convolutional layers of the network extract features from the image while the fully connected layers predict the output probabilities and coordinates. This network architecture is inspired by the GoogLeNet model. This network has twenty four convolutional layers, followed by two fully connected layers. Instead of the inception modules used by GoogLeNet, this one simply uses $1 \times 1$ reduction layers followed by $3 \times 3$ convolutional layers, as presented in Figure. 2.7.

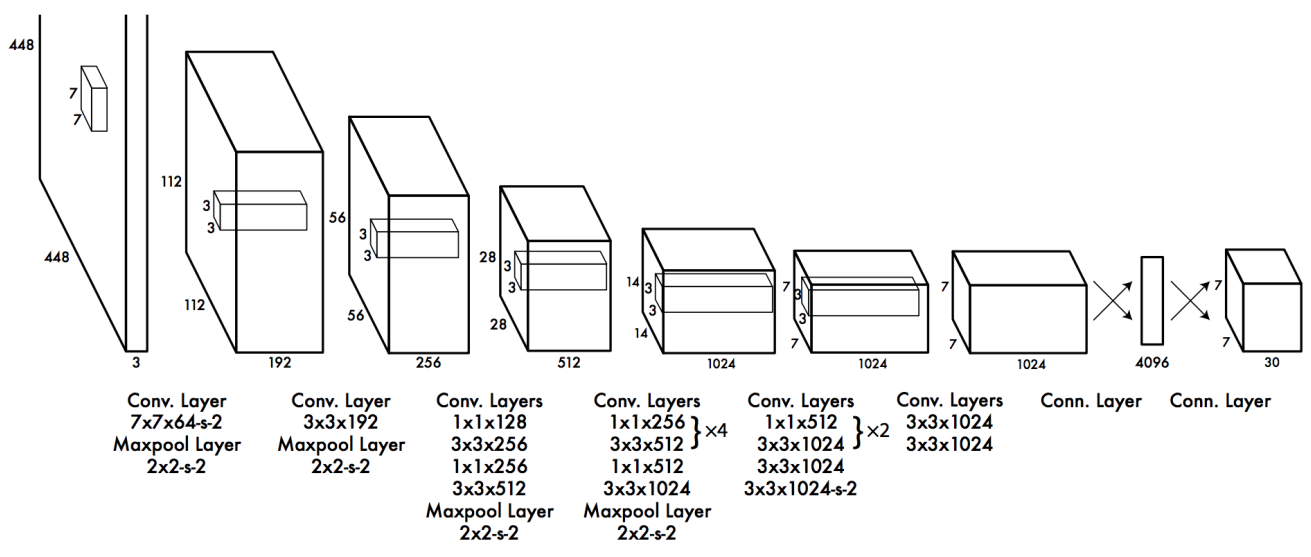

Figure 2.7: YOLO's network architecture

There is fast version of YOLO designed to push the boundaries of fast object detection that uses a neural network with nine convolutional layers, instead of twenty four, and fewer filters in those layers. Other than the size of the network, all training and testing parameters are the same between YOLO and Fast YOLO.

\subsubsection{Training}

For pre-training, the first twenty convolutional layers were used followed by an average-pooling layer, and a fully connected layer. Then to convert the model to perform detection, it was added four convolutional layers and two fully connected layers with randomly initialized weights. The final layer predicts both class probabilities and bounding box coordinates, and normalizes the bounding box width and height by the image width and height so that they fall between 0 and 1 . The sum-squared error method is used in the output of this model because it is simpler to optimize, even though it is not perfect for maximizing average precision. Sum-squared error equally weights errors in large boxes and small boxes, this error metric should reflect that small deviations in large boxes matter less than in small boxes.

During training the loss function is also optimized, the loss function only penalizes classification error if an object is present in that grid cell. It also only penalizes bounding box coordinate 
error if that predictor is responsible for the ground truth box. The network is trained for about 135 epochs. Throughout training a batch size of 64 , a momentum of 0.9 and a decay of 0.0005 were used. This network training recipe was: In the first epochs, the learning rate is slowly raised from 0.001 to 0.01 , training continues with 0.01 for 75 epochs, then 0.001 for 30 epochs, and finally 0.0001 for 30 epochs. To avoid overfitting it is used dropout and extensive data augmentation. A dropout layer with rate 0.5 after the first connected layer prevents co-adaptation between layers. For data augmentation it is introduced random scaling and translations of up to $20 \%$ of the original image size.

\subsubsection{Summary}

Fast YOLO is the fastest real-time detector with $155 \mathrm{fps}$ and a mAP of $52.7 \%$ while YOLO has the best mAP (64.4\%) in the real-time detectors list. When referring to less than real-time detectors YOLO is the fastest with $21 \mathrm{fps}$ and an mAP of $66.4 \%$, but loses in precision to Faster R-CNN VGG-16 that has a mAP of $73.2 \%$ with 7 fps. The technique used by YOLO is interesting for this thesis proposed solution because it is supposed to scale it to a real-time context. Similar solutions, both in results and architecture to YOLO are SSD (Single Shot Detector) [43], and an improved version of YOLO in [44].

\subsubsection{RetinaNet}

Current state-of-the-art object detectors are based on a two-stage, proposal-driven mechanism, just like R-CNN and its variants. Despite the success of these methods, one stage detectors have emerged, such as YOLO. RetinaNet [45] is a one-stage detector that identifies class imbalance during training as the main obstacle impeding a one-stage detector from achieving state-of-the-art accuracy and proposes a new loss function.

\subsubsection{Focal Loss}

The Focal Loss is designed to address the one-stage object detection scenario in which there is an extreme imbalance between foreground and background classes during training. Focal Loss is introduced with cross entropy (CE) loss for binary classification. A notable property of this loss, which can be easily seen in its plot, is that even examples that are easily classified incur a loss with non-trivial magnitude. When summed over a large number of easy examples.

Focal Loss Defnition The author proposes to reshape the loss function to down-weight easy examples and thus focus training on hard negatives. The are two notable properties of the focal loss: (1) When an example is misclassified and the probability is small, the modulating factor is near 1 and the loss is unaffected, but as the probability increases to 1, the factor goes to 0 and the loss for well-classified examples is own-weighted. (2) The focusing parameter smoothly adjusts the rate at which easy examples are down-weighted. When focusing parameter (FP) is zero, focal loss is equivalent to $\mathrm{CE}$, and as FP is increased, the effect of the modulating factor is likewise 
increased. The modulating factor reduces the loss contribution from easy examples and extends the range in which an example receives low loss.

Class Imbalance and Model Initialization Binary classification models are by default initialized to have equal probability of outputting either -1 or 1 . With this initialization, in the presence of class imbalance, the loss due to the frequent class can dominate total loss and cause instability in early training. To counter this, the authors introduced the concept of a prior for the value of probability estimated by the model for the rare class at the start of training. It was found that this improves training stability for both the cross entropy and focal loss in the case of heavy class imbalance.

\subsubsection{RetinaNet Detector}

RetinaNet is a single, unified network composed of a backbone network and two task-specific subnetworks, as depicted in Figure. 2.8. The backbone is responsible for computing a convolutional feature map over an entire input image. The first subnet performs convolutional object classification on the backbone's output. The second subnet performs convolutional bounding box regression. The two subnetworks feature a simple design that the authors propose specifically for one-stage, dense detection.

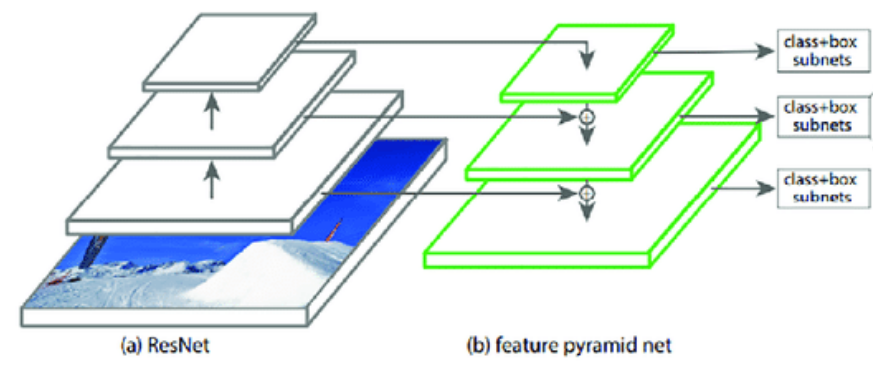

Figure 2.8: RetinaNet overview

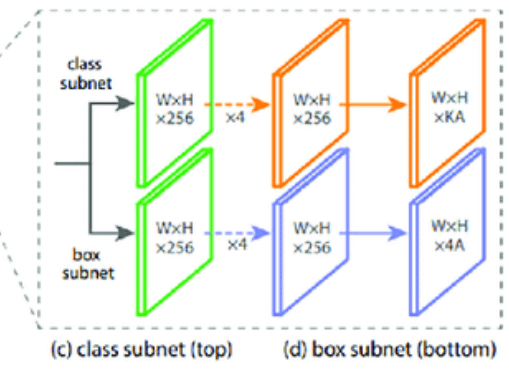

Feature Pyramid Network Backbone The Feature Pyramid Network [46] (FPN) is the backbone network for RetinaNet. FPN augments a standard convolutional network with a top-down pathway, and lateral connections so the network efficiently constructs a rich, multi-scale feature pyramid from a single resolution input image. Each level of the pyramid can be used for detecting objects at a different scale, and it was built on top of the ResNet architecture.

Anchors The anchors have areas of $32^{2}$ to $512^{2}$ on pyramid levels 3 to 7 , respectively. In total there are 9 anchors per level and across levels they cover the scale range 32-813 pixels with respect to the network's input image. Each anchor is assigned a length $K$, one-hot vector of classification targets, where $K$ is the number of object classes, and a 4-vector of box regression targets. Specifically, anchors are assigned to ground-truth object boxes using an intersection-overunion threshold of 0.5. As each anchor is assigned to at most one object box, the corresponding 
entry in its length $K$ label vector is set to 1 and all other entries to 0 . Box regression targets are computed as the offset between each anchor and its assigned object box.

Classification Subnet In this subnet the probability of object presence at each spatial position is predicted for each of the anchors and object classes. Taking an input feature map with $C$ channels from a given pyramid level, the subnet applies four $3 \times 3$ convolutional layers, each with $C$ filters and each followed by ReLU activations, followed by a $3 \times 3$ convolutional layer with $K$ filters. This object classification subnet uses $3 \times 3$ convolutions, and does not share parameters with the box regression subnet.

Box Regression Subnet The design of the box regression subnet is identical to the classification subnet except that it terminates in $4 A$ linear outputs per spatial location. For each of the $A$ anchors per spatial location, these 4 outputs predict the relative offset between the anchor and the groundtruth box. It works In parallel with the object classification subnet but use separate parameters.

\subsubsection{Training}

Focal Loss The loss on the output of the classification is the focal loss developed by the authors. When training RetinaNet, the focal loss is applied to all anchors in each image, the total focal loss is computed a the sum of the focal loss of all the anchors normalized by the number of anchors assigned to a ground-truth box. It is noted that the weight assigned to the rare class should be decreased as the focusing parameter increases.

Initialization ResNet-50 and 101 were experimented as backbones and pre-trained on ImageNet1k, using models released by [31] and initialized as in [46]. All convolutional layers are initialized with bias $b=0$ and a gaussian weight fill with standard deviation of 0.01 . For the final

convolutional layer of the classification subnet the bias was initialized as $b=-\log \left(\frac{1-\pi}{\pi}\right)$. The authors use $\pi=0.01$ in all experiments, preventing the background anchors from generating a large loss value in the first iteration of training.

Optimization RetinaNet is trained with stochastic gradient descent(SGD). Using synchronized SGD in 8 GPUs with sixteen images per minibatch. All models are trained for 90k iterations with an initial learning rate of 0.01 , which is divided by 10 at the $60 \mathrm{k}$ iteration and divided again at the 80k iteration. Horizontal image flipping is used as the only form of data augmentation, weight decay of 0.0001 and a momentum of 0.9. Training time ranges between 10 and 35 hours.

\subsubsection{Summary}

RetinaNet was evaluated on the COCO dataset [40] and compared with the results of state-ofthe-art solutions including both one-stage and two-stage detectors. Regarding one-stage detectors, RetinaNet is faster and gets a superior mAP than DSSD [47] with a gap of 5.9\%. Regarding 
two stage detectors the gap is $2.3 \%$ compared with the top performer Faster R-CNN [48]. This solution identifies the class imbalance as the primary obstacle of one-stage detector and introduces the focal loss that applies a modulating term to the cross entropy loss in order to focus learning on hard negatives. This is interesting for the proposed solution due to the lack of background control of our dataset. 


\section{Chapter 3}

\section{Labeled Waste in the Wild Dataset}

Although several waste datasets have been proposed in the literature, many issues remain unexplored: (i) there are no datasets available that contain different types of waste in the same image; (ii) most of the available datasets were recorded in a very controlled scenario; (iii) there are no waste datasets that were acquired with different sensors.

The dataset presented attempts to address all of these problems. The created Labeled Waste in the Wild dataset (LWW) contains 1002 images of used food trays using several different smartphones. Depending on the application, a real-world setting has a significant weight on the performance of a model. In this manner, this dataset is composed by images from different shopping centers, canteens or home used trays with no control over lighting, background, objects position and type. The images are $3456 \times 4608$, RGB, taken inside building, with good artificial light conditions. LWW dataset was enlarged with 180 images from the Ciocca et al [49] food dataset. Afterwards, images were annotated on Labelbox.com. Illustrative examples are included in Figure. A.1.
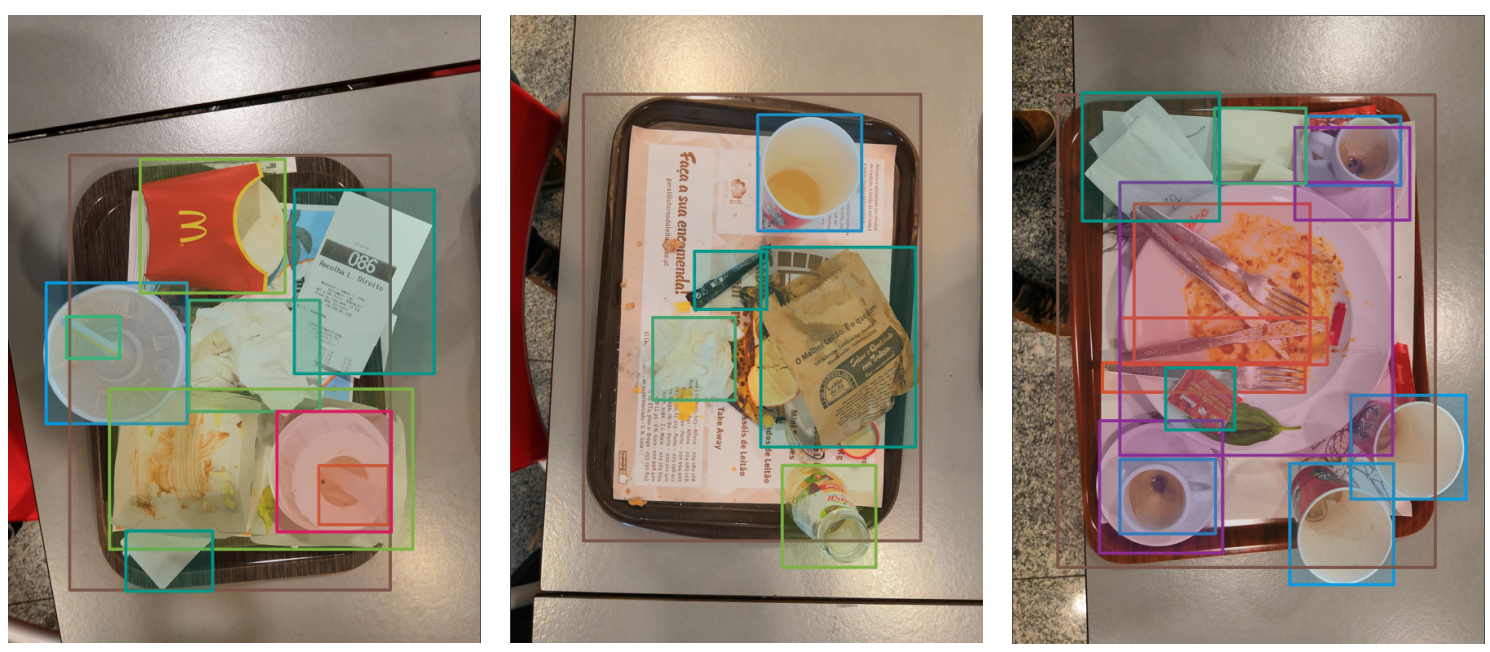

Figure 3.1: Illustrative images and respective annotations. 


\subsection{Classes and Multi-label perspective}

The dataset is composed of 19 different classes totaling 7216 labels. All classes represent a shape and the material they contain as we can see in Table 3.1.

Table 3.1: Frequency of each class in the dataset.

$\begin{array}{llll}\text { bottle_glass } & 26 & \text { bottle_plastic } & 266 \\ \text { box_paper } & 255 & \text { box_plastic } & 54 \\ \text { can_metal } & 112 & \text { cup_glass } & 185 \\ \text { cup_paper } & 423 & \text { cup_plastic } & 225 \\ \text { cutlery_metal } & 703 & \text { cutlery_plastic } & 114 \\ \text { mixed_waste } & 249 & \text { paper_napkin } & 1086 \\ \text { plastic_waste } & 207 & \text { plate_ceramic } & 932 \\ \text { plate_glass } & 18 & \text { plate_plastic } & 124 \\ \text { plastic_straw } & 173 & \text { tray } & 997 \\ \text { paper_paper } & 1067 & \text { total } & 7216\end{array}$

There is an average of 7 objects per image, with a mean $1211 \times 1201$ bounding box size. It is important to note that not every class represents waste. This is due to the fact that in the real world usage, not everything encountered on a tray is waste. Therefore, it is vital for the model not to get confused/mistaken by a glass cup, for instance.

In many robotic applications it is important to establish boundaries to limit the robot action, and that is the purpose of the class tray. The dataset was made available to the general public. The objective is it can be used outside the waste sorting category, perhaps on studies involving fastfood restaurants and their most used objects. Regarding types of waste, for example, the difference between the class paper_paper and paper_napkin is the presence of organic waste in it, a piece of relevant information for industrial recycling.

Multi-label is a characteristic of this annotation once the labels can be rearranged in two groups of classes, either by shape or material, and we use that to our advantage in the object detection and classification. The annotation can be framed in a multi-label setting, where each object is annotated in respect to the shape and to the material (and the bounding box). The material label can take values in the set \{glass, paper, metal, plastic\}. The shape label can take values in the set $\{$ cup, plate, box, tray, cutlery, mixed_waste, bottle, paper, can, plastic $\}$.

\subsection{Cross-Sensor application}

Labeled Waste in the Wild contains 400 more images for cross-sensor validation of algorithms. The images are composed of photos of two different sets containing 100 used food trays. Each set has two images of the same tray using different smartphone cameras. The first set was obtained using an Honor 10 and a Xiaomi Redmi Note 3, and the second set was obtained using the Honor 10 and Iphone 5. The images were labeled in the same conditions as the original 1002 images of the dataset. 


\subsection{Summary}

In a real-world application, not every object is considered waste. For that reason in this dataset a significant portion of the objects are not waste. It is vital that our model learns to distinguish variety. The lack of control in the dataset enables more robustness of an algorithm for a realworld application. The multi-label perspective of the dataset allows different approaches on the architecture of a model, increasing the spectrum of results for the objectives proposed, but also to study the behaviour of the different architectures and apply that knowledge in other categories of object recognition. 


\section{Chapter 4}

\section{Hierarchical Deep Detection and Classification of Waste}

Consider a set of images of waste in food trays. The goal is, in each image, to detect the bounding boxes of all the waste objects present, and assign each detected object to one of the considered classes.

A standard baseline approach is presented in section 4.1, followed by an improvement using a cascaded flat model described in section 4.2. The final section 4.3, details the hierarchical model based in two different approaches, based on shape and material.

\subsection{Standard baseline approach: Flat method}

The standard baseline approach consists on building a model that uses all images and detects for each input simultaneously the bounding box and the waste class. This approach is the typical deep learning approach in which all efforts are supported by the neural network and, usually, has positive results. In order to select a model to serve as a base to the architecture, three state-ofthe-art models in object detection and classification are tested, Yolov3 [44], Retinanet [45] and Faster R-CNN [24]. Since these models resize all images to a fixed, low spatial resolution (600 pixels), for computational efficiency, a lot of information is lost when resizing the images from their $3456 \times 4608$ original size. 


\subsection{Improved baseline approach: Cascaded Region Proposal and Clas- sification}

After analyzing the results of the flat method, Faster R-CNN was adopted. To overcome the spatial resolution issue, in this method Faster R-CNN is again trained in the flat semantic label space of 19 classes, but we only keep the proposed bounding boxes. In a second step, using the Bounding box coordinates in the original image, the original image is cropped and resized to $500 \times 500$, and fed as input to a CNN for (flat) classification. The advantage is that this CNN is with images with more detail than present in Faster R-CNN, enabling better classification accuracy. The described architecture is reflected in Figure. 4.1.

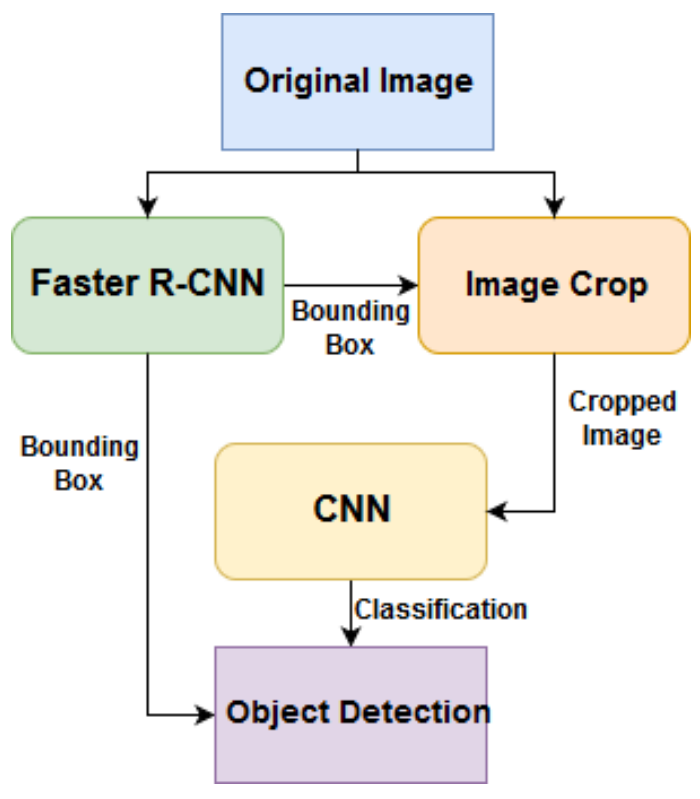

Figure 4.1: Cascaded Region Proposal and Classification

\subsubsection{CNN Development}

A better processing time and accuracy made convolutional neural networks (CNN) prominent for image classification using deep learning techniques. For object detection deep layered CNNs have been used obtaining satisfactory results. In the model proposed, deep CNNs are used to obtain the bounding box of the object. For classification, a conventional CNN has just as few layers, enabling faster processing time without a significant loss in accuracy.

The first architecture, inspired by work tested on MNIST Fashion Dataset [50], is an eightlayer neural network. The input is a RGB image of size $500 \times 500$, followed by two twodimensional convolutional layers with kernel size of $3 \times 3$ and Relu as activation function, then a Max Pooling layer comes into action, having a pool size of $2 \times 2$, to avoid neuron dependency a dropout layer is added with $25 \%$ of the neurons being dropped, a flatten layer is added to allocate the features in a vector and feed a Dense layer activated with Relu, to prevent overfitting 
another dropout layer is added, this time with $50 \%$ of the neurons dropped, the final layer using the classifier softmax to output the predicted class. This description is depicted in Figure. 4.2.

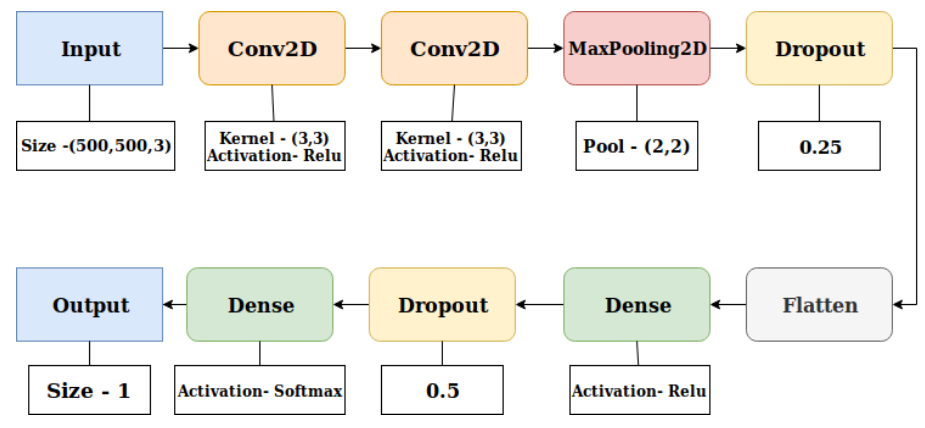

Figure 4.2: CNN Architecture 1.

The results were not satisfactory. Therefore, a second attempt was in order, with the objective of obtaining more features for a more accurate classificaton, an architecture based on the CNN depicted in Figure. 4.2, was developed. A third two-dimensional convolutional layer, two batch normalization layers and an activation layer was added. In contrast, the max pooling layer was removed. The input, with the same size, feeds the first convolutional layer, with kernel size of $3 \times 3$, without an activation, the batch normalization layer comes after to prevent overfitting, followed by the activation function Relu, the second convolutional layer is simililar to the first, the difference being that it is activated by Relu. For the same reason as before, a batch normalization layer is placed, the third convolutional layer follows with equal kernel size and activation function to the first layer. The final layers resemble the layers of the architecture 1, a dropout layers is added, followed by the flatten layer that feeds the vector to the first dense layer, to prevent overfitting, another dropout layer is placed where $50 \%$ of the neurons are dropped, finally the dense layer, activated by the classifier softmax, outputs the class predicted. The architecture is shown in Figure. 4.3.

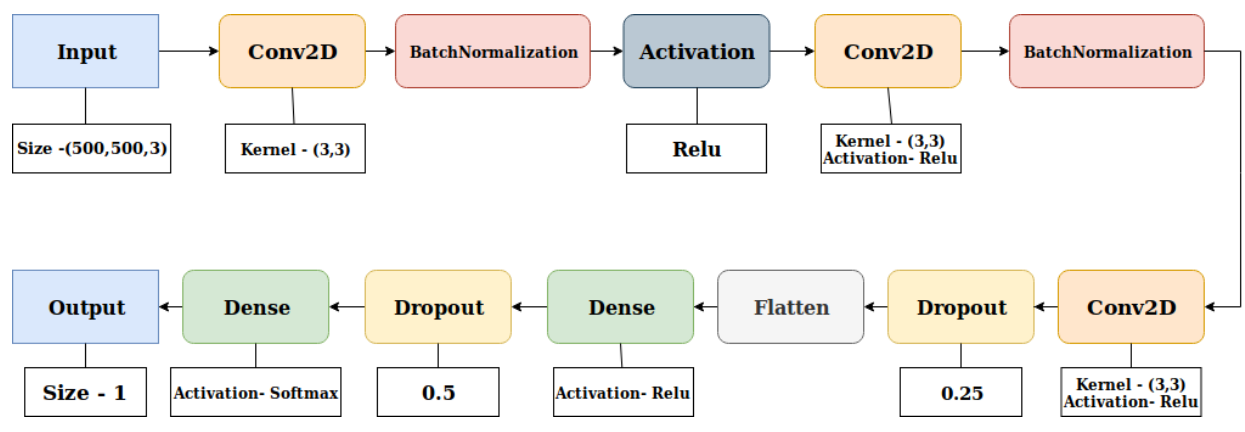

Figure 4.3: CNN Architecture 2.

Architecture 2 is an eleven-layered neural network, compared to architecture 1, is significantly heavier, taking longer to train and to process information. The size-accuracy trade off is not favorable as the accuracy score is not reasonable. The third architecture was developed on top of the knowledge obtained after testing the two previous architectures. 
Being proven that an architecture with fewer layers has better chances of obtaining a reasonable result, a convolutional, batch normalization and dropout layers were removed, and the max pooling layer added. An RGB image of size $500 \times 500$ is the input to a two-dimensional convolutional layer, with kernel size of $3 \times 3$. The activation function comes after the batch normalization layer, and it is the linear rectifier, Relu, feeding another convolutional layer with the same kernel size and activation function. A max pooling layer follows reducing the sample size and transforming it on a vector by the flatten layer, feeding a dense layer activated by Relu. To reduce the neuron dependency, a droput layer is added and 50\% of the neurons are dropped. The output is given by the final dense layer activated by the softmax classifier. This architecture, seen in Figure. 4.4, reached reasonable accuracy results and was adopted as the CNN for the improved baseline and hierarchical approaches.

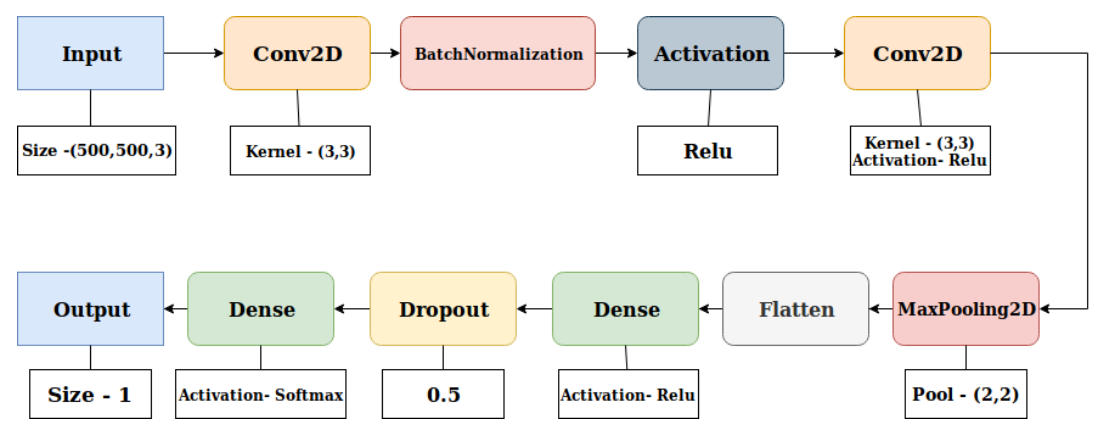

Figure 4.4: CNN Architecture 3.

\subsection{Hierarchical approach}

The rationale for the proposed two-step hierarchical deep learning approach is to divide the semantic classification problem in two subproblems. In a first step, Faster R-CNN is used as informative region proposal, we keep the bounding box and the classification, a mother-class based on shape or material, taking advantage of the multi-label characteristic of the LWW dataset as mentioned in chapter 3. In a second step, the original image is cropped, resized to $500 \times 500$ and fed to the CNN, with the same architecture detailed in Figure. 4.4, trained for the specific mother-class, outputting a child-class based on shape or material. Splitting the hierarchical approach into shape and material was inspired by the work produced by Geirhos et al [51].

\subsubsection{Material based method}

As aforementioned, Faster R-CNN is trained for 4 mother-classes based on material \{glass, paper, metal, plastic\}. Four CNNs are trained, one for each mother-class, to prevent the detection of a class that does not exist. For example, if the mother class is metal then we know that the child class cannot be a cup.

This model receives an image as input, then the Faster R-CNN outputs the bounding box and the mother-class. With that information, the original image is cropped, which is the input of the 
CNN related to the mother-class. Next outputs the child-class, which merged with the motherclass, forms the final class. The latter, with the bounding box predicted before forms the detection of the model. The structure of the mentioned model can be seen in Figure 4.5.

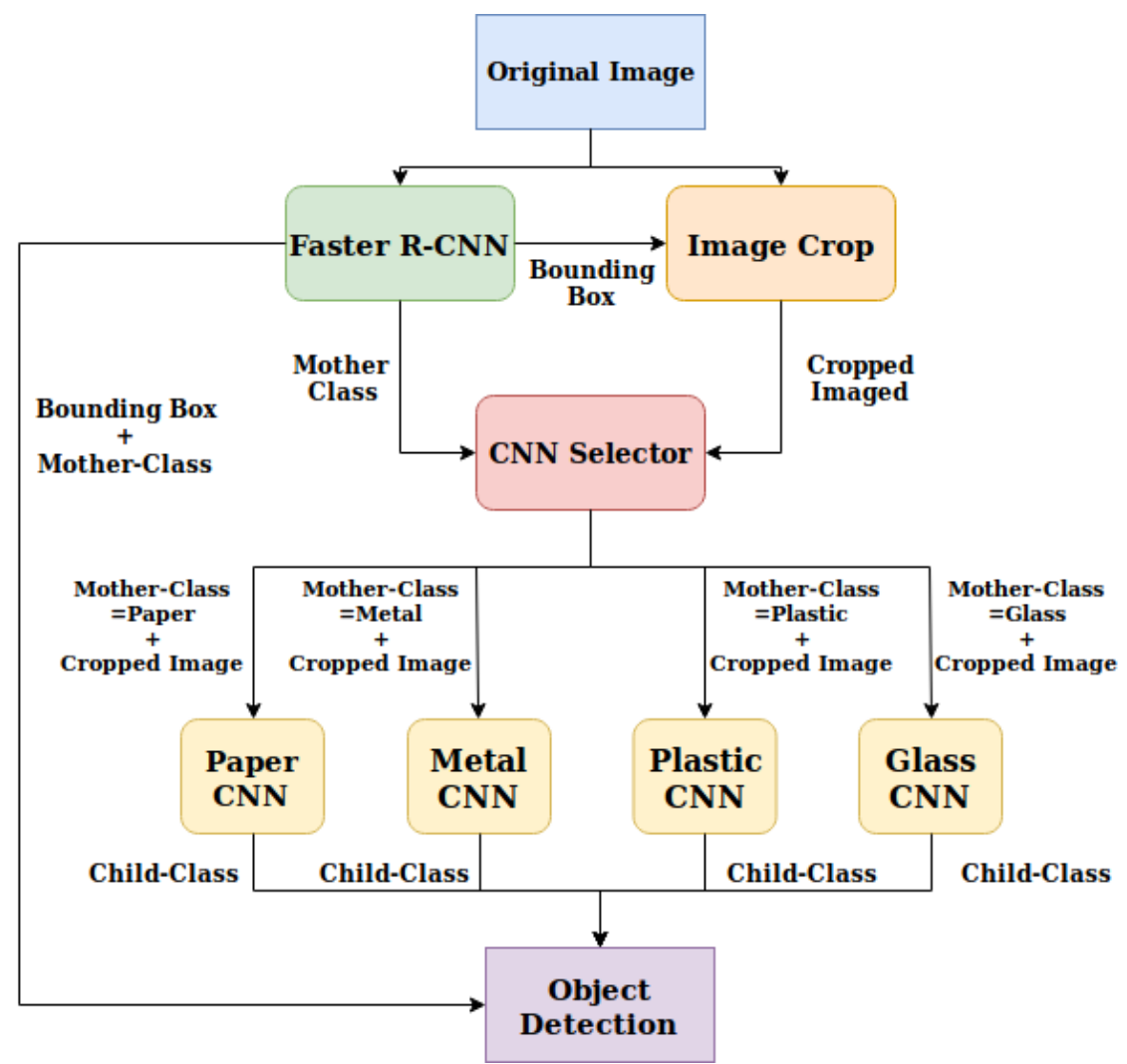

Figure 4.5: Material Based Hierarchical Approach

\subsubsection{Shape based method}

In this method, Faster R-CNN is trained for 10 mother-classes based on their shape \{cup, plate, box, tray, cutlery, mixed_waste, bottle, paper, can, plastic\}. Some mother-classes are unique and do not need a CNN associated with them. If the output of the Faster R-CNN is tray, mixed_waste or can, then they are the final class.

Seven CNNs are trained for the same reason mentioned in section 4.3.1, this time if the motherclass is a cup, it is impossible for the child class to be metal This model behaves very similarly to the material based one, as detailed in Figure. 4.6, an image is used as input to the Faster R-CNN that outputs a bounding box and a mother-class. In this case it is a shape. An image, result of the cropped original image using the bounding box coordinates, is the input of the CNN. This outputs a child class, a material, and together the final class and bounding box correspond to the detection of the model. 


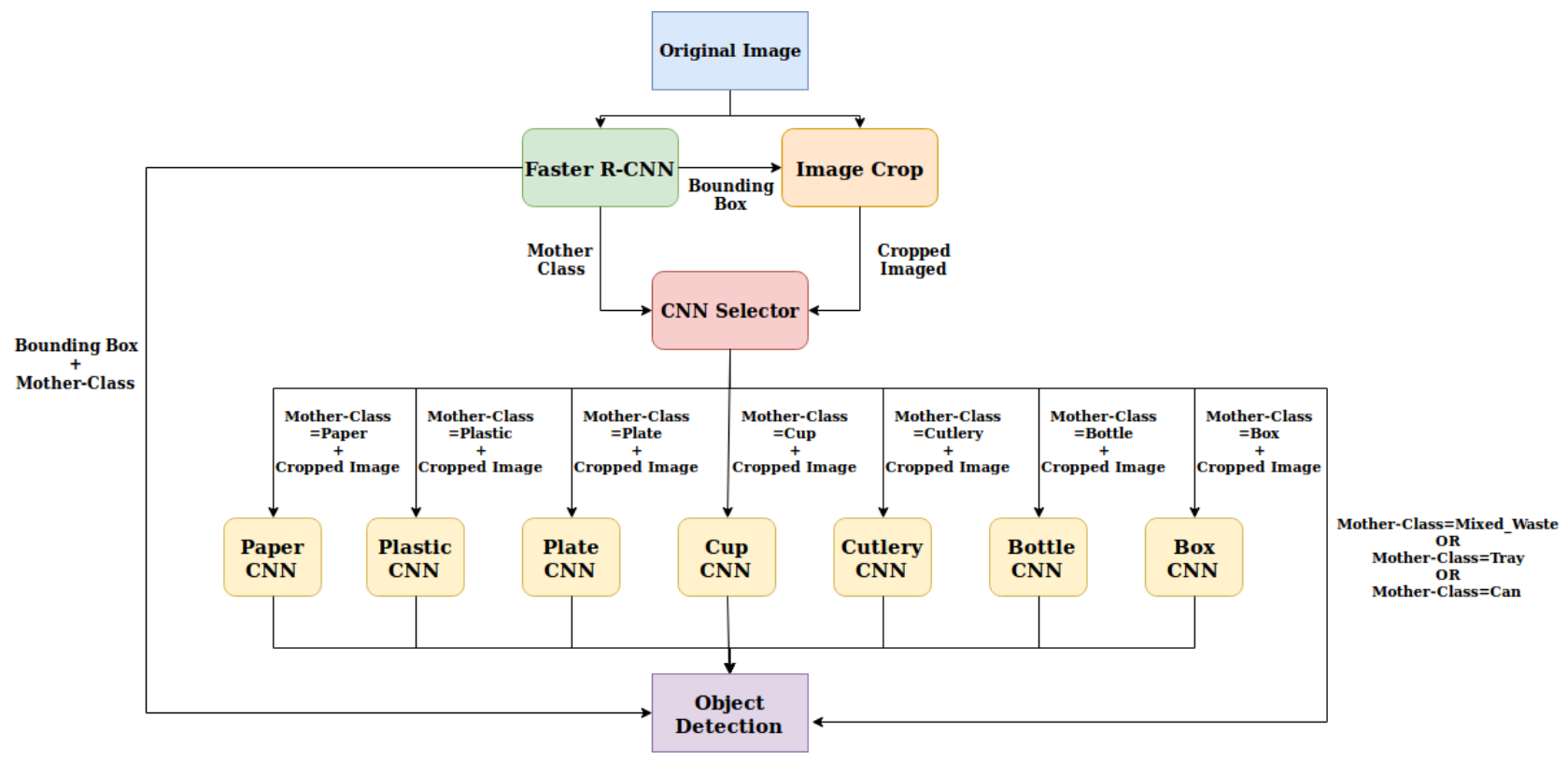

Figure 4.6: Shape Based Hierarchical Approach 


\section{Chapter 5}

\section{Experimental Study}

In this chapter the experimental results are described. The performance evaluating metrics are presented in section 5.1. The test on the three state of the art models is detailed on section 5.2, followed by the experiments on the cascaded model in section 4.1,also containing the tests of the different $\mathrm{CNN}$ architectures. In section 5.4 the two hierarchical methods' tests are explained and results are drawn. Cross-sensor validation is portrayed in section 5.5. Results are analyzed and discussed in section 5.6.

Important to note a common element in all tests is the use of the LWW dataset, the same 800 images were used to train the models. For testing, with the exception of the cross-sensor validation, the same 200 images were used.

\subsection{Metrics}

The metric of evaluation used on the model is the mean average precision (mAP), its purpose is the evaluation of state-of-the-art algorithms in object detection and classification. The mAP definition used is the same as the PASCAL VOC Challenge [36]. This evaluation method is algorithm independent, meaning that any model that outputs a bounding box and classification can be evaluated by this method. The method can be segregated in two independent steps, the first one being the calculation of the intersection over union, and the second the average precision calculation.

\subsubsection{Intersection over Union}

To calculate the intersection over union (IoU), two inputs are necessary, the bounding box predicted by the model ( $\mathrm{Pbb}$ ) and the ground-truth bounding box (GTbb). A bounding box is a set of coordinates, corresponding to a box that involves an object in a given image. The score of the IoU ranges from 0 to 1 , and it is calculated by dividing the area of intersection of the bounding boxes, over the area of union of the same bounding boxes, as shown in Equation. 5.1.

$$
I o U=\frac{P b b \cap G T b b}{P b b \cup G T b b}
$$




\subsubsection{Average Precision}

The second step of the mAP calculation is the average precision score (AP). Having the classification and the IoU score, it is possible to determine if a detection is a true positive (TP), a false positive (FP) or a False Negative (FN). According to the PASCAL VOC Challenge, a detection is a true positive if the classification is correct and the IoU is higher then 0.5. A false positive if the IoU is again higher than 0.5 but with a wrong classification, and a false negative if the object is present in the image, but the model does not predict any bounding box, or if the IoU is lower than 0.5. These data enables the calculation of Precision and Recall. Precision (Equation. 5.2), is calculated by dividing the number of true positives over the sum of true positives and false positives. Recall (Equation. 5.3), is calculated by dividing the number of true positives over the sum of true positives and false negatives.

$$
\begin{aligned}
& \text { Precision }=\frac{\text { TruePositives }}{\text { TruePositives }+ \text { FalsePositives }} \\
& \text { Recall }=\frac{\text { TruePositives }}{\text { TruePositives }+ \text { FalseNegatives }}
\end{aligned}
$$

The next step is the calculation of the Precision/Recall curve, for that the interpolated precision is obtained, as shown in Equation. 5.4, by interpolating the precision at each recall level $r$, taking the maximum precision measured for a class when the corresponding recall surpasses $r$.

$$
\operatorname{Pinterp}(r)=\max _{\tilde{r}: \tilde{r} \geq r} p(\tilde{r})
$$

The advantage in interpolating the precision/recall curve is the reduction of the impact of "ripple" caused by small variations in the ranking. Finishing this step, the average precision is defined by the area under the curve, and is calculated by the mean precision at a set of eleven equally segregated recall levels 0,0.1,0.2,0.3,..., 1 , as depicted in Equation. 5.5.

$$
A P=\frac{1}{11} \sum_{r \in\{0,0.1,0.2, \ldots, 1\}} \operatorname{Pinterp}(r)
$$

The average precision is calculated for each class, the mAP is then obtained by calculating the mean of the average precision, as shown in Equation. 5.6.

$$
m A P=\frac{1}{Q} \sum_{q}^{Q} A P(q)
$$

Where $Q$ is the number of classes, $q$ a specific class and the $A P(q)$ the average precision of the given class $q$. 


\subsection{Standard baseline approach - Flat method}

Three state-of-the-art models, Yolov3, Retinanet and Faster R-CNN, were trained and tested in the flat semantic labels of the Labeled Waste in the Wild dataset. The objective is to select one of the models as a backbone of the hierarchical architecture. All three models were trained on the same 800 images. Due to the different nature of the models the training parameters were different. Yolo was trained for 3000 iterations, with a batch size of 32, corresponding to 120 epochs during 25 hours. On the other hand, Faster R-CNN had 1000 iterations per epoch, was trained for 120 epochs for a duration of 40 hours. Finally, Retinanet was trained for 12 epochs, each with 10000 iterations for a duration of 48 hours. Neither of the models was trained with pre-trained weights, causing longer training time. The models were tested on the same 200 images and results, shown in Table 5.1, demonstrated that Faster R-CNN had the best mAP score, being adopted as the backbone of the developed architecture.

Table 5.1: Flat method - mAP score

\begin{tabular}{ll}
\hline Model & $\mathrm{mAP}(\%)$ \\
\hline Yolov3 & 33.2 \\
Retinanet & 71.4 \\
Faster R-CNN & 74.1 \\
\hline
\end{tabular}

\subsection{Cascaded Region Proposal and Classification}

In this test, Faster R-CNN was used as a region proposal network, taking advantage of the training for the baseline flat approach, only the predicted bounding boxes were kept. The classification task is left to a developed CNN. The data to train and test the CNNs was obtained by cropping the images using the bounding box coordinates from the ground truth labels, resulting in 7216 labeled images of objects.

\subsubsection{CNN performance}

Three different CNN architectures, described in Chapter. 4, were subject to testing to find a reasonable solution to the classification task. For each architecture, two different optimizers were used, an adaptive optimizer, Adam [52], and the Stochastic Gradient Descent (SGD) [53]. In Wilson et al [54], the authors claim that in deep learning algorithms, SGD has a better training performance than adaptive methods, including Adam. The parameters used for each optimizer were the default values, recommended by the authors, seen in Table. 5.2. 
Table 5.2: Optimizer's hyperparameters

\begin{tabular}{llllllll}
\hline Optimizer & Learning Rate & Beta_1 & Beta_2 & Epsilon & Momentum & Nesterov & Decay \\
\hline Adam & 0.001 & 0.9 & 0.999 & $10^{-8}$ & - & - & 0 \\
SGD & 0.001 & - & - & - & 0.9 & True & $10^{-6}$ \\
\hline
\end{tabular}

From the 7216 images obtained after cropping the ground truth labels, 6400 were randomly chosen for training, and 800 for testing. Some data augmentation techniques were used, such as changing brightness, zooming in and flipping the images, in order to increase the size of the training dataset. Training and Validation performance, such as, loss (L) and accuracy (ACC), are detailed in Table 5.3. The loss function used was the categorical cross entropy, and the batch size was fixed to 16.

Table 5.3: Architecture/Optimizer Performance

\begin{tabular}{lllllll}
\hline CNN & Optimizer & Data Augmentation & Training $_{L}$ & Validation $_{L}$ & Training $_{A C C}$ & Validation $_{A C C}$ \\
\hline Architecture 1 & Adam & Flip, Brightness, Zoom & 14.1 & 13.7 & $12.4 \%$ & $15 \%$ \\
Architecture 1 & SGD & Flip, Brightness, Zoom & 0.31 & 4.3 & $88.5 \%$ & $47.5 \%$ \\
\hline Architecture 2 & Adam & Flip, Brightness, Zoom & 12.5 & 13.8 & $14.3 \%$ & $14.3 \%$ \\
Architecture 2 & SGD & Flip, Brightness, Zoom & 13.6 & 13.7 & $13.3 \%$ & $15.0 \%$ \\
\hline Architecture 3 & Adam & Flip, Brightness, Zoom & 14.24 & 13.4 & $11.3 \%$ & $17.0 \%$ \\
Architecture 3 & SGD & Flip, Brightness, Zoom & 0.37 & 1.6 & $87.2 \%$ & $67.0 \%$ \\
\hline
\end{tabular}

Several conclusions are drawn from the test. Architecture 3 presents the best results regarding overffiting and validation accuracy, proving that adding a batch normalization layer improves the training of the model. SGD presents better results in all architectures, confirming the claims in the Wilson et al [54] study. Adding a third convolutional layer to the model (architecture 2) worsened results.

The CNN adopted for the task of classification of the cascaded method is based on the Architecture 3, trained using SGD. Before integration, and because the learning rate with SGD is the same through out the training, three more tests were done, comparing the Architecture 3 performance with learning rate of $0.01,0.001$ and 0.0001. Results shown in Table 5.4.

Table 5.4: Architecture/Learning Rate Performance

\begin{tabular}{lllllll}
\hline CNN & Optimizer & Learning Rate & Training $_{L}$ & Validation $_{L}$ & Training $_{A C C}$ & Validation $_{A C C}$ \\
\hline Architecture 3 & SGD & 0.01 & 0.44 & 1.9 & $85.4 \%$ & $62.5 \%$ \\
Architecture 3 & SGD & 0.001 & 0.37 & 1.6 & $87.2 \%$ & $67.0 \%$ \\
Architecture 3 & SGD & 0.0001 & 0.29 & 2.04 & $90.4 \%$ & $62.3 \%$
\end{tabular}

Architecture 3 with the SGD optimizer and a learning rate of 0.001 proved to have the best performance, and is adopted to the integrated model and for the Hierarchichal model. 


\subsubsection{Integrated model performance}

In Fig. 4.1, the integration between Faster R-CNN and the CNN is depicted. Faster R-CNN trained in the flat semantic labels outputing a bounding box, that boundig box used to crop the original image, resizing it to $500 \times 500$ and feeding it to the CNN, Architecture 3, seen in Fig. 4.4, outputing the class that joined to the bounding box, forms the detection. Tested on the 200 test images of the LWW dataset, the mAP score of this model reached $81.4 \%$, a clear improvement of the $74.1 \%$ mAP score of the Faster R-CNN standard base line approach.

\subsection{Hierarchical Model}

The Cascaded Region Proposal and Classification method improved the mAP score in 7.3\%, proving that images with more detail help in the task of classification. Taking that rational further, the hierarchical method was designed, the difference is that Faster R-CNN outputs not only a bounding box, but also a mother-class. The advantage of the CNN in having an image with more detail is kept, but the task is limited to a classification of shape or material, depending on the mother-class, given by Faster R-CNN. The model is more accurately described in Section 4.3.

\subsubsection{Material Based}

Faster R-CNN was trained in the material semantic labels of the LWW dataset, corresponding in 4 mother-classes (Paper, Plastic, Metal and Glass). The training process was similar to previous testing, the same 800 images were used for training. The model was trained for 120 epochs with 1000 iterations per epoch, for approximately 40 hours. Using the same 200 images for testing, the mAP score obtained for the 4 mother-classes, and before integration with the CNNs, was $72.8 \%$.

Each mother-class has one $\mathrm{CNN}$ dedicated, meaning that in this architecture, four CNNs were trained, to output a child class based on shape. CNN_paper is the CNN linked to the motherclass paper, trained for 4 child-classes (box, paper, napkin and cup). CNN_plastic is linked to the mother-class plastic, trained for 9 child-classes (bottle, cutlery, mixed_waste, plastic_waste, straw, cup, tray, plate and box). CNN_glass linked to the mother-class glass, trained for 4 child-classes (bottle, cup, plate_glass and plate_ceramic). CNN_metal corresponding to the mother-class metal, trained for 2 child-classes (can and cutlery). The four CNNs have the same architecture, represented in Fig. 4.4, and equal to the one used in the cascaded region proposal and classification. Images to train the CNN were obtained by cropping the images from the LWW dataset, using the bounding box coordinates of the shape semantic ground truth labels. The same data augmentation techniques used to train the $\mathrm{CNN}$ of the Cascaded method were reused, such as changing brightness, zooming in and flipping the images, in order to increase the size of the training dataset. All CNNs trained for 100 epochs with the SGD optimizer and a learning rate of 0.0001. In Table 5.5, more details, such as the accuracy reached, the number of images used for training and testing, are presented. 
Table 5.5: Hierarchichal Method - Material Based - CNN training results

\begin{tabular}{lllll}
\hline CNN & $\mathrm{n}^{0}$ child-classes & $\mathrm{n}^{0}$ training images & $\mathrm{n}^{\mathrm{o}}$ testing images & Testing Accuracy \\
\hline CNN_Paper & 4 & 2272 & 560 & $42 \%$ \\
CNN_Plastic & 9 & 1920 & 480 & $67 \%$ \\
CNN_Metal & 2 & 640 & 160 & $84 \%$ \\
CNN_Glass & 4 & 928 & 224 & $78 \%$ \\
\hline
\end{tabular}

The integration of the Faster R-CNN with these CNNs is depicted in Fig. 4.5. The images used for testing were the same 200 images of the LWW dataset used in previous tests. The mAP score of this model was $80.9 \%$.

\subsubsection{Shape Based}

Faster R-CNN was trained in the shape semantic labels of the LWW dataset, corresponding in 10 mother-classes (Paper, Plastic, Plate, Cup, Cutlery, Bottle, Box, Mixed_Waste, Tray and Can). The training process was equal to the Material based process. The same 800 images were used for training. The model was trained for 120 epochs with 1000 iterations per epoch, for approximately 40 hours. Using the same 200 images for testing, the mAP score obtained for the 10 motherclasses, and before integration with the CNNs, was $73.6 \%$.

In the shape based method not every mother-class has one CNN dedicated. Tray, Mixed_Waste and Can are unique, in the sense that in the LWW dataset there are only cans made out of metal, meaning that if the mother class is Can, Tray or Mixed_Waste then Faster R-CNN is responsible for the object detection, without the involvement of a CNN. In this architecture, 7 CNNs were trained, to output a child class based on material. CNN_paper is the CNN linked to the mother-class paper, trained for 2 child-classes (paper and napkin). CNN_plastic is linked to the mother-class plastic, trained for 2 child-classes (plastic_waste and straw). CNN_bottle linked to the mother-class bottle, trained for 2 child-classes (glass and plastic). CNN_box corresponding to the mother-class box, trained for 2 child-classes (paper and plastic). CNN_cup is linked to the mother-class cup, trained for 3 child-classes (plastic, paper and glass). The mother-class cutlery has a link to the CNN_cutlery, trained for 2 child-classes (plastic and metal). Finally, CNN_plate is linked to the mother-class plate, trained for 3 child-classes (plastic, ceramic and glass). The seven CNNs have the same architecture, represented in Fig. 4.4, and equal to the one used in the cascaded and the hierarchical material based methods. The methodology of obtaining the training data was similar to the one used in the material based method, where the images were obtained by cropping the original images from the LWW dataset, using the bounding box coordinates, in this case, of the material semantic ground truth labels. The same data augmentation techniques used to train the CNNs of the Cascaded and Material based methods were reused, such as changing brightness, zooming in and flipping the images. Following the training pattern used before, all CNNs trained for 100 epochs with the SGD optimizer and a learning rate of 0.0001. In Table 5.6, 
more details, such as the accuracy reached, the number of images used for training and testing, are presented.

Table 5.6: Hierarchichal Method - Material Based - CNN training results

\begin{tabular}{lllll}
\hline CNN & $\mathrm{n}^{\mathrm{o}}$ child-classes & $\mathrm{n}^{\mathrm{o}}$ training images & $\mathrm{n}^{\mathrm{o}}$ testing images & Testing Accuracy \\
\hline CNN_Paper & 2 & 1728 & 416 & $75 \%$ \\
CNN_Plastic & 2 & 288 & 80 & $73 \%$ \\
CNN_Bottle & 2 & 224 & 64 & $90 \%$ \\
CNN_Box & 2 & 240 & 64 & $84 \%$ \\
CNN_Cup & 3 & 672 & 160 & $72 \%$ \\
CNN_Cutlery & 2 & 656 & 160 & $89 \%$ \\
CNN_Plate & 3 & 832 & 240 & $87 \%$ \\
\hline
\end{tabular}

The integration of the Faster R-CNN with these CNNs is depicted in Fig. 4.6. The images used for testing were the same 200 images of the LWW dataset used in previous tests. The mAP score of this model was $86.0 \%$. Detection examples using this model can be found in the appendix A.

\subsection{Cross-Sensor Validation}

Out of all the models tested, the hierarchical method based on shape achieves the best performance. The 200 images of the LWW dataset used to test the models were obtained using the same camera (Honor 10 smartphone). The cross-sensor validation method consists in testing the same model on images of the same trays obtained with different cameras, hence the name "cross-sensor". The LWW dataset was designed to enable cross-sensor validation, apart from the 1002 images, 400 more images were added. From those 400 images, two different sets of 100 trays are present, from each set, two different cameras took the photos, resulting in 200 images of each set. From the first set, an Honor 10 and a Xiaomi Redmi Note 3 were used, and in the second set, an Honor 10 and an Iphone 5 were used. The mAP score of the hierarchical shape based model are present in Table. 5.7.

Table 5.7: Cross-Sensor Validation mAP score

\begin{tabular}{lll}
\hline Set & sensor & mAP $(\%)$ \\
\hline 1 & Honor 10 & 90.7 \\
1 & Xiaomi Redmi Note 3 & 92.9 \\
\hline 2 & Honor 10 & 88.6 \\
2 & Iphone 5 & 84.0 \\
\hline
\end{tabular}

Each set has the same trays and objects, the only difference being in the sensor. Having this information into account, results show that the sensor has influence on the performance of the algorithm. Honor 10, the most used sensor on the LWW dataset has a worse perfomance than Xiaomi Redmi Note 3, but a better performance than Iphone 5. The fact that the mAP is 
calculated on 100 images has the disadvantage of some classes having only a few representations in the dataset, influencing the individual AP of that class and ultimately, influencing the mAP score.

\subsection{Discussion}

\subsubsection{Model Performance Overview}

The experimental study revealed test on four different methodologies, a standard baseline approach, a cascaded region proposal and classification, and two hierarchical methods, one based on shape and another based on material. The mAP scores of these models are summarized in Table 5.8.

Table 5.8: Model Results

\begin{tabular}{ll}
\hline Model & $\mathrm{mAP}(\%)$ \\
\hline Flat & 74.1 \\
Cascaded & 81.4 \\
Hierarchical - Material & 80.9 \\
Hierarchical - Shape & $\mathbf{8 6}$ \\
\hline
\end{tabular}

Differences are noticeable when comparing the flat and cascaded approach, the latter improved the mAP in $7.3 \%$. Faster R-CNN resizes the images to a maximum of 600 pixels on height or width, that causes loss of information on our $3456 \times 4608$ images. This improves the duration of training and prediction with a good performance when the classes have significant differences. Moreover, there is a huge difference between an airplane, a car and a person, making possible to correctly classify the objects with fewer features. However that is not the case when the objective is to distinguish between a cup of paper, a cup of glass or a cup of plastic. Intuitively, if an image has a lower resolution some details will be lost, making the classification of Faster R-CNN harder. In order to overcome this limitation, our CNN uses the bounding box coordinates predicted by Faster R-CNN re-scaled to the original size to crop the original image, overcoming the resolution problem, proving improved results on complex classes.

The hierarchical method based on shape obtained the best result, compared to the flat approach, improved the mAP in $11.9 \%$, and compared to the hierarchical method based on material the improvement reached $5.1 \%$. The difference between the two hiearchical approaches is a matter of complexity. As seen in Table 5.9, Faster R-CNN does not get significant mAP changes with the different number of classes. 
Table 5.9: Label Semantic mAP score

\begin{tabular}{llll}
\hline Model & Label Semantic & $\mathrm{n}^{\mathrm{o}}$ of Classes & $\mathrm{mAP}(\%)$ \\
\hline Faster R-CNN & Flat & 19 & 74.1 \\
Faster R-CNN & Material & 4 & 72.8 \\
Faster R-CNN & Shape & 10 & 73.6 \\
\hline
\end{tabular}

The complexity matter is significant on the CNN level. The architecture used for the CNN (Fig. 4.4) is a nine layered CNN, advantageous for several reasons, the simplicity enables the integration with Faster R-CNN on off-the-shelf hardware, the largest training time was three hours, taken by the CNN trained for 19 classes, which is relatively quick when compared to the 40 hours training time of Faster R-CNN. However, the more classes it has to distinguish, the lower the performance. Table 5.10 demonstrates a relation between the mean number of classes and the mean accuracy of the CNNs used in the cascaded region proposal and the to hierarchical methods.

Table 5.10: Mean $\mathrm{n}^{\mathrm{o}}$ of classes/accuracy of the CNNs

\begin{tabular}{lll}
\hline CNN & Average $\mathrm{n}^{0}$ of Classes & Average Accuracy (\%) \\
\hline Cascaded RP and Classification & 19 & 67.0 \\
Hierarchical - Material Based & 4.75 & 67.8 \\
Hierarchical - Shape Based & 2.29 & 81.4 \\
\hline
\end{tabular}

The CNNs of the hierarchical based on material, and of the cascaded region proposal and classification have similar mean accuracy, reflecting in a difference of $0.5 \%$ in the map score. However, when the classification task of the adopted CNN is almost binary, as is the case in the hierarchical shape based method, the average accuracy drastically improves, hence the difference in the mAP score. Another possibility is the fact that the CNNs of the hierarchical shape based method classify based on material. Material has more texture in common than shape, in Geirhos et al. [51], the authors claim that CNN tend to focus more on texture than shape.

\subsubsection{Influence of Less Represented Classes}

The mAP is literally the mean of the individual average precision (AP) of each class. The influence of less represented classes is not relevant for the comparison between models due to the fact that every model was tested on the same images with the same ground truth labels. But it means that the absolute value of the mAP score might be optimistic. Tables 5.11 and 5.12 show the individual AP scores of each class in the four tested models.

Looking at the tables, less represented classes, such as cutlery_plastic, plate_glass, box_plastic, cup_glass, plastic and can, clearly benefit from the CNN classification with detailed images, proving that Faster R-CNN has problems learning on complex classes with low resolution. The flat model has lower performance on most of the classes when compared to the best model, hierarchical based on shape. The latter having a poor AP on the class plate_ceramic, an out-liar, 
Table 5.11: Individual AP - Part I

\begin{tabular}{lllllllllll}
\hline Model & $\begin{array}{l}\text { AP } \\
\text { tray }\end{array}$ & $\begin{array}{l}\text { AP } \\
\text { plate_ceramic }\end{array}$ & $\begin{array}{l}\text { AP } \\
\text { napkin }\end{array}$ & $\begin{array}{l}\text { AP } \\
\text { bottle_plastic }\end{array}$ & $\begin{array}{l}\text { AP } \\
\text { cup_paper }\end{array}$ & $\begin{array}{l}\text { AP } \\
\text { cutlery_metal }\end{array}$ & $\begin{array}{l}\text { AP } \\
\text { bottle_glass }\end{array}$ & $\begin{array}{l}\text { AP } \\
\text { paper }\end{array}$ & $\begin{array}{l}\text { AP } \\
\text { cup_glass }\end{array}$ & $\begin{array}{l}\text { AP } \\
\text { cup_plastic }\end{array}$ \\
\hline $\begin{array}{l}\text { Flat } \\
\text { Cascaded }\end{array}$ & 0.97 & 0.87 & 0.68 & 0.48 & 0.76 & 0.75 & 0.74 & 0.55 & 0.63 \\
$\begin{array}{l}\text { Hierarchical - } \\
\text { Material }\end{array}$ & 0.92 & 0.92 & 0.91 & 0.85 & 0.66 & 0.47 & 0.8 & 0.67 & 1 & 0.74 \\
$\begin{array}{l}\text { Hierarchical - } \\
\text { Shape }\end{array}$ & 0.99 & 0.62 & 0.71 & 0.58 & 0.22 & 1 & 0.8 & 1 & 0.83 & 0.83 \\
\hline
\end{tabular}

Table 5.12: Individual AP - Part II

\begin{tabular}{|c|c|c|c|c|c|c|c|c|c|}
\hline Model & $\begin{array}{l}\text { AP } \\
\text { plastic }\end{array}$ & $\begin{array}{l}\text { AP } \\
\text { can }\end{array}$ & $\begin{array}{l}\text { AP } \\
\text { box_paper }\end{array}$ & $\begin{array}{l}\text { AP } \\
\text { mixed_waste }\end{array}$ & $\begin{array}{l}\text { AP } \\
\text { straw }\end{array}$ & $\begin{array}{l}\text { AP } \\
\text { plate_plastic }\end{array}$ & $\begin{array}{l}\text { AP } \\
\text { cutlery_plastic }\end{array}$ & $\begin{array}{l}\text { AP } \\
\text { plate_glass }\end{array}$ & $\begin{array}{l}\text { AP } \\
\text { box_plastic }\end{array}$ \\
\hline Flat & 0.68 & 0.57 & 0.46 & 0.9 & 1 & 0.92 & 0.95 & 0.5 & 0.4 \\
\hline Cascaded & 0.78 & 0.94 & 0.72 & 0.6 & 0.94 & 0.97 & 1 & 1 & 1 \\
\hline $\begin{array}{l}\text { Hierarchical - } \\
\text { Material }\end{array}$ & 0.75 & 1 & 1 & 0.22 & 0.63 & 0.86 & 1 & 1 & 1 \\
\hline $\begin{array}{l}\text { Hierarchical - } \\
\text { Shape }\end{array}$ & 0.86 & 0.88 & 0.75 & 0.85 & 1 & 1 & 1 & 1 & 1 \\
\hline
\end{tabular}

demonstrating the poor performance of the $\mathrm{CNN}$ in distinguishing between ceramic, plastic and glass. Overall, fewer represented classes have higher AP, influencing the absolute value of the mAP, but there the individual AP values legitimize the hierarchical shape based model as the best option.

\subsubsection{Understanding Cross-Sensor Validation}

The cross-sensor validation test was done on half of the total images used for testing the models. On section 5.6.2, the influence of less represented classes is discussed, and the knowledge is that, when analyzing the mAP scores of each sensor in its set, the comparison between absolute value of the mAP of the model on the different sets and testing images is discouraged. For that reason, further analysis on individual AP of classes of each set of trays is done. Tables 5.13 and 5.14 represent the individual AP of classes of set 1, where the Honor 10 and the Xiaomi Redmi Note 3 are tested.

Table 5.13: Individual AP Set 1 - Part I

\begin{tabular}{|c|c|c|c|c|c|c|c|c|c|c|}
\hline Model & $\begin{array}{l}\mathrm{AP} \\
\text { tray }\end{array}$ & $\begin{array}{l}\text { AP } \\
\text { plate_ceramic }\end{array}$ & $\begin{array}{l}\text { AP } \\
\text { napkin }\end{array}$ & $\begin{array}{l}\text { AP } \\
\text { bottle_plastic }\end{array}$ & $\begin{array}{l}\text { AP } \\
\text { cup_paper }\end{array}$ & $\begin{array}{l}\text { AP } \\
\text { cutlery_metal }\end{array}$ & $\begin{array}{l}\text { AP } \\
\text { bottle_glass }\end{array}$ & $\begin{array}{l}\text { AP } \\
\text { paper }\end{array}$ & $\begin{array}{l}\text { AP } \\
\text { cup_glass }\end{array}$ & $\begin{array}{l}\text { AP } \\
\text { cup_plastic }\end{array}$ \\
\hline Honor 10 & 0.99 & 0.99 & 0.65 & 0.79 & 0.98 & 0.63 & 1 & 0.75 & 1 & 0.86 \\
\hline Xiaomi & 0.99 & 0.97 & 0.60 & 1 & 0.97 & 0.88 & 1 & 0.82 & 0.96 & 0.76 \\
\hline
\end{tabular}

Table 5.14: Individual AP Set 1 - Part II

\begin{tabular}{|c|c|c|c|c|c|c|c|c|c|}
\hline Model & $\begin{array}{l}\text { AP } \\
\text { plastic }\end{array}$ & $\begin{array}{l}\text { AP } \\
\text { can }\end{array}$ & $\begin{array}{l}\text { AP } \\
\text { box_paper }\end{array}$ & $\begin{array}{l}\text { AP } \\
\text { mixed_waste }\end{array}$ & $\begin{array}{l}\text { AP } \\
\text { straw }\end{array}$ & $\begin{array}{l}\text { AP } \\
\text { plate_plastic }\end{array}$ & $\begin{array}{l}\text { AP } \\
\text { cutlery_plastic }\end{array}$ & $\begin{array}{l}\text { AP } \\
\text { plate_glass }\end{array}$ & $\begin{array}{l}\text { AP } \\
\text { box_plastic }\end{array}$ \\
\hline Honor 10 & 1 & 1 & 0.86 & 0.82 & 1 & - & 1 & 1 & 1 \\
\hline Xiaomi & 1 & 1 & 0.94 & 0.88 & 1 & - & 1 & - & 1 \\
\hline
\end{tabular}


Honor 10 is the sensor that captured the majority of images used to train the model, but Xiaomi has a better mAP score. The fact that the test was made with 100 images might explain the $2.2 \%$ difference in mAP score. Some classes had very low or none representation, plate_plastic is not present in set 1, and plate_glass has so few entries that the model tested on Xiaomi was not able to detect that class. There is not a major difference on most classes. With more images it's estimated that the mAP score would get more similar and closer to the original score obtained on the 200 test images of the LWW dataset. Regarding set 2, some differences were noticed between the Honor 10 and the Iphone 5. Tables 5.15 and 5.16 represent the individual AP of classes of set 2.

Table 5.15: Individual AP Set 2 - Part I

\begin{tabular}{|c|c|c|c|c|c|c|c|c|c|c|}
\hline Model & $\begin{array}{l}\mathrm{AP} \\
\text { tray }\end{array}$ & $\begin{array}{l}\text { AP } \\
\text { plate_ceramic }\end{array}$ & $\begin{array}{l}\text { AP } \\
\text { napkin }\end{array}$ & $\begin{array}{l}\text { AP } \\
\text { bottle_plastic }\end{array}$ & $\begin{array}{l}\text { AP } \\
\text { cup_paper }\end{array}$ & $\begin{array}{l}\text { AP } \\
\text { cutlery_metal }\end{array}$ & $\begin{array}{l}\text { AP } \\
\text { bottle_glass }\end{array}$ & $\begin{array}{l}\text { AP } \\
\text { paper }\end{array}$ & $\begin{array}{l}\text { AP } \\
\text { cup_glass }\end{array}$ & $\begin{array}{l}\text { AP } \\
\text { cup_plastic }\end{array}$ \\
\hline Honor 10 & 0.99 & 0.94 & 0.76 & 0.42 & 0.92 & 0.75 & 1 & 0.75 & 1 & 0.96 \\
\hline Iphone 5 & 0.53 & 0.56 & 0.59 & 73 & 0.85 & 0.62 & 1 & 0.74 & 0.96 & 0.94 \\
\hline
\end{tabular}

Table 5.16: Individual AP Set 2 - Part II

\begin{tabular}{llllllllll}
\hline Model & $\begin{array}{l}\text { AP } \\
\text { plastic }\end{array}$ & $\begin{array}{l}\text { AP } \\
\text { can }\end{array}$ & $\begin{array}{l}\text { AP } \\
\text { box_paper }\end{array}$ & $\begin{array}{l}\text { AP } \\
\text { mixed_waste }\end{array}$ & $\begin{array}{l}\text { AP } \\
\text { straw }\end{array}$ & $\begin{array}{l}\text { AP } \\
\text { plate_plastic }\end{array}$ & $\begin{array}{l}\text { AP } \\
\text { cutlery_plastic }\end{array}$ & $\begin{array}{l}\text { AP } \\
\text { plate_glass }\end{array}$ & $\begin{array}{l}\text { AP } \\
\text { box_plastic }\end{array}$ \\
\hline Honor 10 & 1 & 1 & 0.64 & 0.76 & 0.94 & 1 & 0.75 & 1 \\
Iphone 5 & 1 & 0.94 & 0.68 & 0.8 & 1 & 1 & 1 & 1 & 1 \\
\hline
\end{tabular}

There is large difference in the tray and plate_ceramic AP scores, and in most of the classes the Honor 10 has a better performance, the Iphone has a better score on bottle_plastic and cutlery_plastic, and the performance is similarly high on less represented classes. For an unknown reason the Iphone has a poor performance, probably a result of the post-processing that Apple's IOS preforms on photos.

Cross-Sensor Validation proves to be a significant test for deep learning models, helping developers understand the behaviour of the models and testing the robustness for a real-world application. 


\section{Chapter 6}

\section{Conclusions}

This chapter is divided into three sections. Firstly, in section 6.1, objectives of the dissertation are reviewed and analyzed in context with the knowledge produced. Advantages and limitations of the contributions are scrutinized in section 6.2. Lastly, section 6.3 includes future developments.

\subsection{Research Objectives}

The five objectives introduced in chapter 1 guarantee, if accomplished, valuable contributions to the scientific community. Four of the objectives were completed, in chapter 3 a novel dataset is introduced, achieving the uncontrolled real-world setting, with an acceptable amount of multilabeled images, accomplishing the first outlined objective. In chapter 5, precisely in section 5.2, the standard baseline approach test is described, the intention behind the test was to chose the backbone of the hierarchical model. For that reason three state of the art models, with different architectural ideologies, were tested under similar conditions, completing the second objective. Chapter 4 presents the different architectures developed and the rationale behind their design. The chosen model in the standard baseline approach was scaled to a cascaded model, the latter being scaled to an hierarchical method with two different semantics. The developed model is not restricted to a fixed number of classes, images or context, fulfilling the third objective. Experimental results are discussed in section 5.6, hypothesising possible reasons, with the aid of detailed test data, for the improvement of the developed models relative to the state of the art models, concluding the forth objective. The fifth and last objective was partially completed. Despite of being trained and having reasonable performance on uncontrolled real-world images, and being tested on different cameras, completing a cross-sensor validation test, the algorithm is not yet designed for a computational-effective application. Being computational-effective is a significant requisite for an industrial solution, at the date of writing is not reasonable to equip sorting plants with hardware capable of processing the algorithm. 


\subsection{Strengths and Weaknesses}

The LWW dataset enables a real-world trained model, due to the uncontrolled lighting, object positioning and type, nature of the images. The ability for a cross-sensor validation facilitates the design of a fully automated machine that uses the algorithm. The number of images in the dataset is scarce, originating difficulties in the training process, a large dataset enables more generalization during training, creating a robust model. About $90 \%$ of the images in the dataset were obtained in Portuguese shopping centers, creating a possible bias on brands commonly found in these establishments.

Regarding the models, the improvement on the mAP score, relative to the flat approach using state of the art solutions, demonstrates that for problems involving complex classes, the use of an hierarchical approach based on shape, that crops the original image, is a viable method to apply on a solution. This along with the LWW dataset are perhaps the most relevant contribution of the thesis. As mentioned in the previous section, the algorithm is yet to be computational-effective, preventing its use on a real-world application. Moreover, if a new class is added, a new CNN needs to be trained and added to the hierarchical model.

\subsection{Future Work}

Suggestions for future work should lay on strengthening some of the weaknesses previously identified, as well as, updating the algorithm in order to use less computational resources. Another possible research line to be developed would be the application of our proposed model on video format, facilitating the use of a robotic system for waste sorting. 


\section{Appendix A}

\section{Detection Examples}
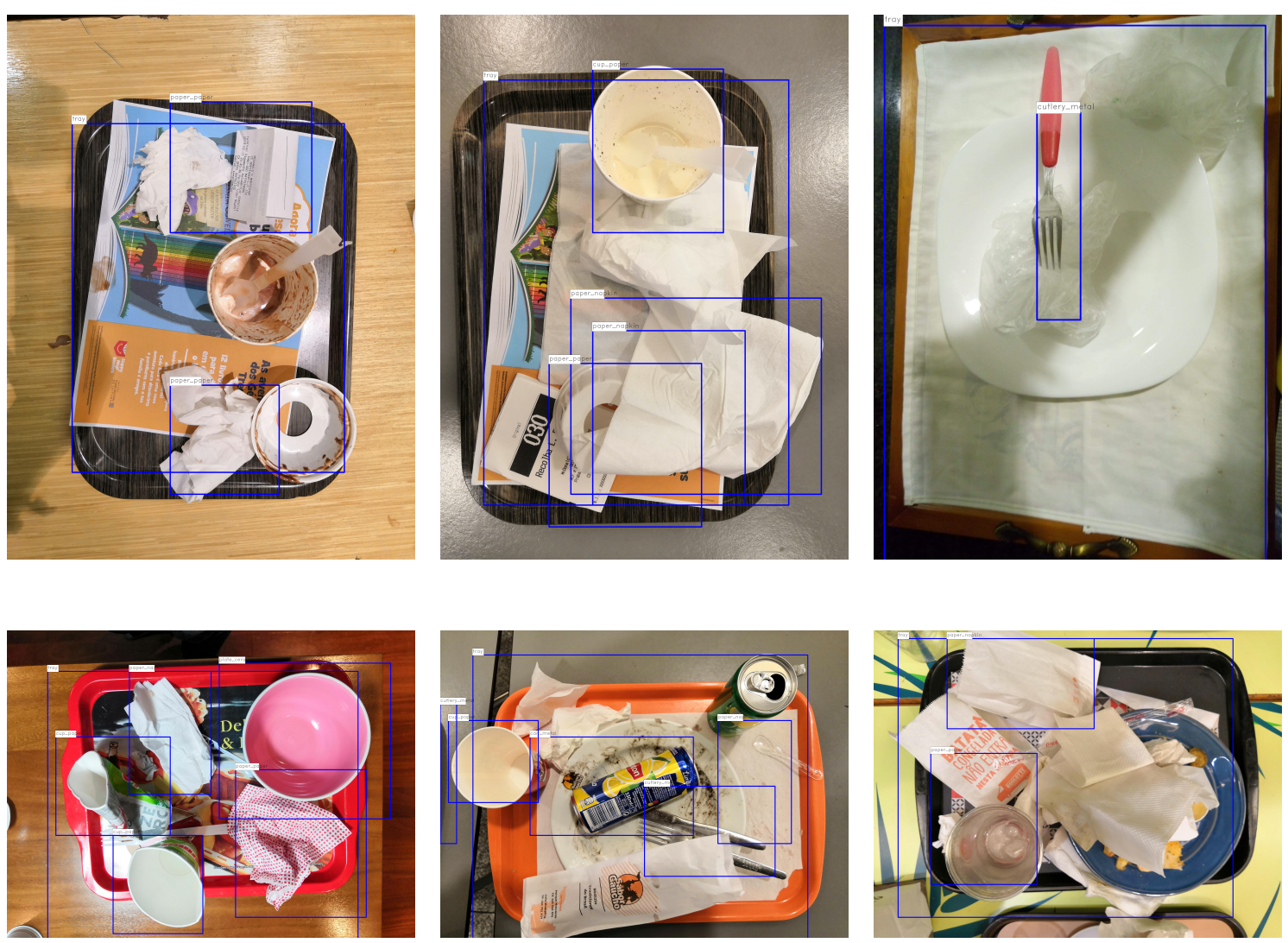

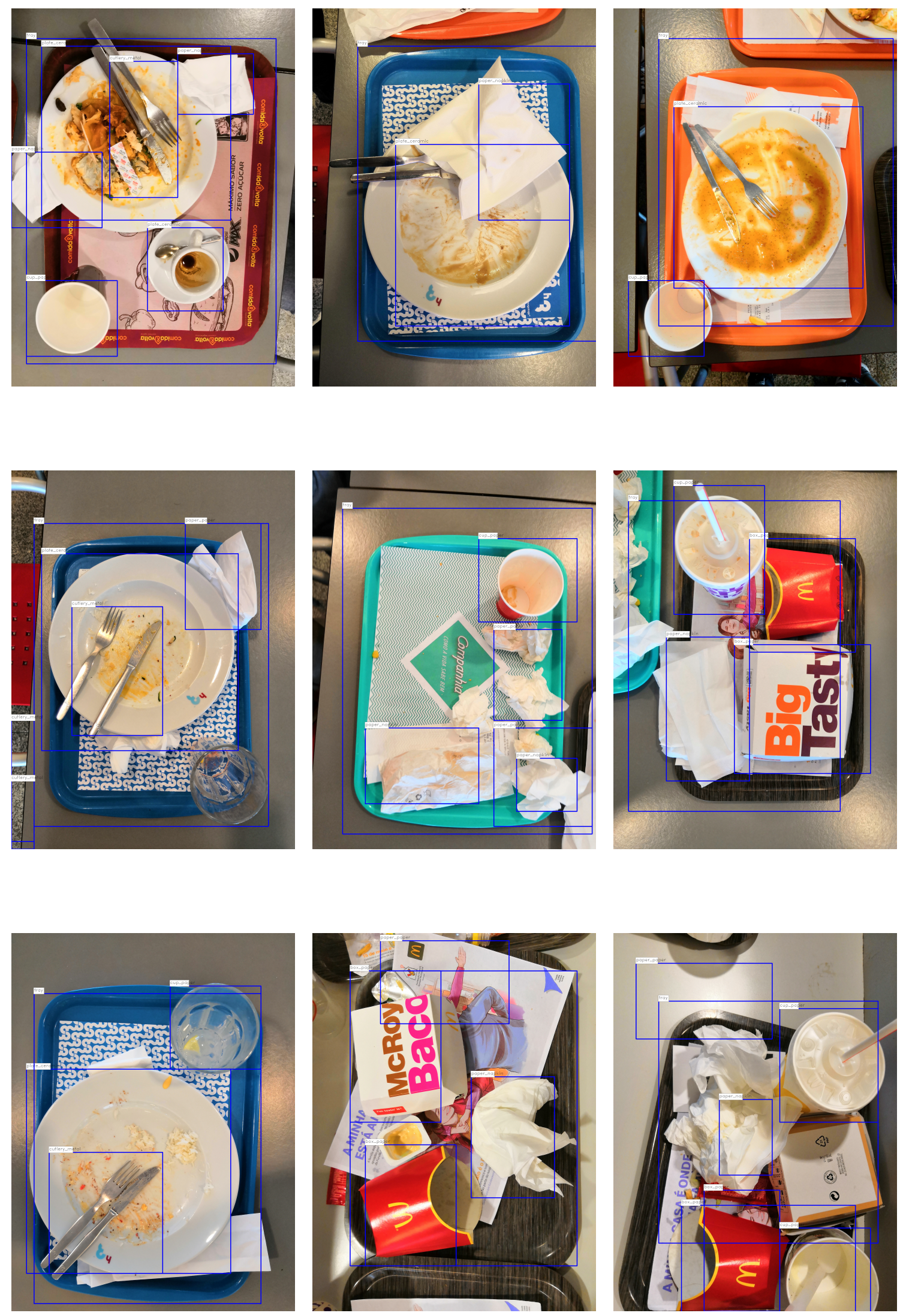

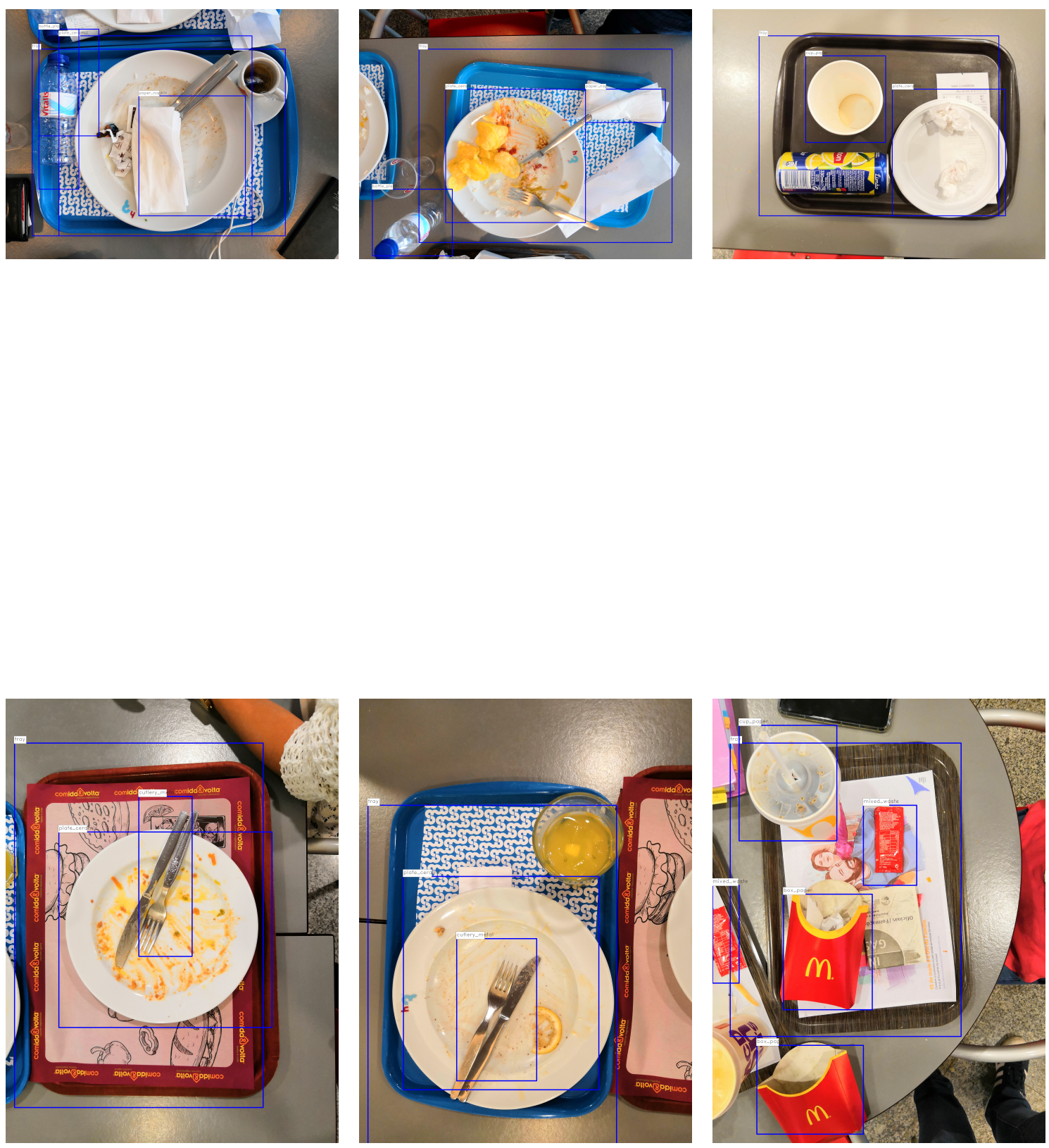

Figure A.1: Hierarchical shape based method - detection examples 


\section{References}

[1] Recycling: How does it work? - solid waste and recycling services. URL: https://recycle.georgetown.org/ georgetown-resource-recovery-and-solid-waste-services/faq/ recycling-how-does-it-work/.

[2] Ahead of general assembly kick-off, un chief urges world leaders to unite in 'time of turmoil' I africa renewal onlinel 2019-0205. URL: https://www.un.org/africarenewal/africaga/news/ ahead-general-assembly-kick-un-chief-urges-world-leaders-unite-\% $\mathrm{E} 2 \% 80 \div 98$ time-turmoil $\% \mathrm{E} 2 \% 80 \% 99$.

[3] Alberto Coentrão. Comissão europeia põe em causa números da reciclagem em portugal. Público, $102018 . \quad$ URL: https://www.publico.pt/2018/10/08/sociedade/noticia/ comissao-europeia-poe-em-causa-numeros-da-reciclagem-em-portugal-1846301.

[4] United Nations. About the sustainable development goals. un.org. URL: https://www . un.org/sustainabledevelopment/sustainable-development-goals/.

[5] D M Scott. Two-colour near-infrared sensor for sorting recycled plastic waste. Measurement Science and Technology, 6:156, 01 1999. doi:10.1088/0957-0233/6/2/004.

[6] European environmental agency - recycling rates in europe. URL: https://www . eea.europa.eu/about-us/competitions/waste-smart-competition/ recycling-rates-in-europe/image_view_fullscreen.

[7] Bhs systems win gold, silver excellence awards - bulk handling systems https://www.bulkhandlingsystems.com/bhs-systems-win-gold-silver-excellenceawards/ - 2019-01-30. URL: https://www.bulkhandlingsystems.com/ bhs-systems-win-gold-silver-excellence-awards/.

[8] Adhithya Prasanna M, S Vikash Kaushal, and P Mahalakshmi. Survey onidentification and classification of waste for efficient disposal and recycling. International Journal of Engineering Technology,7:520, 03 2018. doi:10.14419/ijet.v7i2.8.10513.

[9] Serge Belongie, Jitendra Malik, and Jan Puzicha. Shape matching and object recognition using shape contexts. Technical report, CALIFORNIA UNIV SAN DIEGO LA JOLLA DEPT OF COMPUTER SCIENCE AND ENGINEERING, 2002.

[10] Ron O Dror, Edward H Adelson, and Alan S Willsky. Recognition of surface reflectance properties from a single image under unknown real-world illumination. 2001. 
[11] Ce Liu, Lavanya Sharan, Edward H Adelson, and Ruth Rosenholtz. Exploring features in a bayesian framework for material recognition. In Computer Vision and Pattern Recognition (CVPR), 2010 IEEE Conference on, pages 239-246. IEEE, 2010.

[12] Alex Krizhevsky, Ilya Sutskever, and Geoffrey E Hinton. Imagenet classification with deep convolutional neural networks. In Advances in neural information processing systems, pages 1097-1105, 2012.

[13] Yann Glouche and Paul Couderc. A smart waste management with self-describing objects. In The Second International Conference on Smart Systems, Devices and Technologies (SMART'13), 2013.

[14] Maher Arebey, MA Hannan, Hassan Basri, Rawshan Ara Begum, and Huda Abdullah. Integrated technologies for solid waste bin monitoring system. Environmental monitoring and assessment, 177(1-4):399-408, 2011.

[15] Sadjad ABDOLI. Rfid application in municipal solid waste management system. INTERNATIONAL JOURNAL OF ENVIRONMENTAL RESEARCH (IJER), 2009.

[16] Maher Arebey, MA Hannan, Rawshan Ara Begum, and Hassan Basri. Solid waste bin level detection using gray level co-occurrence matrix feature extraction approach. Journal of environmental management, 104:9-18, 2012.

[17] George E Sakr, Maria Mokbel, Ahmad Darwich, Mia Nasr Khneisser, and Ali Hadi. Comparing deep learning and support vector machines for autonomous waste sorting. In Multidisciplinary Conference on Engineering Technology (IMCET), IEEE International, pages 207-212. IEEE, 2016.

[18] Moacir Antonelli Ponti, Leonardo Sampaio Ferraz Ribeiro, Tiago Santana Nazare, Tu Bui, and John Collomosse. Everything You Wanted to Know about Deep Learning for Computer Vision but Were Afraid to Ask. Proceedings - 2017 30th SIBGRAPI Conference on Graphics, Patterns and Images Tutorials SIBGRAPI-T 2017, 2018-Janua:17-41, 2018. doi:10.1109/SIBGRAPI-T.2017.12.

[19] Corinna Cortes and Vladimir Vapnik. Support-vector networks. Machine learning, 20(3):273-297, 1995.

[20] Jay Donovan. Auto-trash sorts garbage automatically at the techcrunch disrupt hackathon. Techcrunch Disrupt Hackathon, 2016.

[21] Gary Thung Mindy Yang and Gary Thung. Classification of trash for recyclability status. CS229 Project Report, 2016, 2016.

[22] David G Lowe. Distinctive image features from scale-invariant keypoints. International journal of computer vision, 60(2):91-110, 2004.

[23] Oluwasanya Awe, Robel Mengistu, and Vikram Sreedhar. Smart trash net: Waste localization and classification. 2017.

[24] Shaoqing Ren, Kaiming He, Ross Girshick, and Jian Sun. Faster R-CNN: Towards RealTime Object Detection with Region Proposal Networks. IEEE Transactions on Pattern Analysis and Machine Intelligence, 39(6):1137-1149, 2017. arXiv:1506.01497, doi: 10.1109/TPAMI.2016.2577031. 
[25] Bernardo S. Costa, Aiko C. S. Bernardes, Julia V. A. Pereira, Vitoria H. Zampa, Vitoria A. Pereira, Guilherme F. Matos, Eduardo A. Soares, Claiton L. Soares, and Alexandre Silva. Artificial intelligence in automated sorting in trash recycling. pages 198-205, 102018. doi:10.5753/eniac.2018.4416.

[26] Karen Simonyan and Andrew Zisserman. Very deep convolutional networks for large-scale image recognition. arXiv preprint arXiv:1409.1556, 2014.

[27] Cenk Bircanoğlu, Meltem Atay, Fuat Beşer, Özgün Genç, and Merve Ayyüce Kızrak. Recyclenet: Intelligent waste sorting using deep neural networks. In 2018 Innovations in Intelligent Systems and Applications (INISTA), pages 1-7. IEEE, 2018.

[28] Fei-fei Li, Justin Johnson, and Serena Yeung. CS231 Lecture 11 : Detection and Segmentation. Cs 231, 2017.

[29] Shai Shalev-Shwartz and Shai Ben-David. Understanding machine learning: From theory to algorithms, volume 9781107057. 2013. doi:10.1017/CBO9781107298019.

[30] Olga Russakovsky, Jia Deng, Hao Su, Jonathan Krause, Sanjeev Satheesh, Sean Ma, Zhiheng Huang, Andrej Karpathy, Aditya Khosla, Michael Bernstein, Alexander C. Berg, and Li Fei-Fei. ImageNet Large Scale Visual Recognition Challenge. International Journal of Computer Vision (IJCV), 115(3):211-252, 2015. doi:10.1007/s11263-015-0816-y.

[31] Kaiming He, Xiangyu Zhang, Shaoqing Ren, and Jian Sun. Deep residual learning for image recognition. In Proceedings of the IEEE conference on computer vision and pattern recognition, pages 770-778, 2016.

[32] Christian Szegedy, Wei Liu, Yangqing Jia, Pierre Sermanet, Scott Reed, Dragomir Anguelov, Dumitru Erhan, Vincent Vanhoucke, and Andrew Rabinovich. Going deeper with convolutions. In Proceedings of the IEEE conference on computer vision and pattern recognition, pages 1-9, 2015.

[33] Ross Girshick, Jeff Donahue, Trevor Darrell, and Jitendra Malik. Rich feature hierarchies for accurate object detection and semantic segmentation. Proceedings of the IEEE Computer Society Conference on Computer Vision and Pattern Recognition, pages 580-587, 2014. arXiv:1311.2524, doi:10.1109/CVPR.2014.81.

[34] Ross Girshick, Jeff Donahue, Trevor Darrell, and Jitendra Malik. Region-based convolutional networks for accurate object detection and segmentation. IEEE transactions on pattern analysis and machine intelligence, 38(1):142-158, 2016.

[35] J. R.R. Uijlings, K. E.A. Van De Sande, T. Gevers, and A. W.M. Smeulders. Selective search for object recognition. International Journal of Computer Vision, 104(2):154-171, 2013. arXiv:1409.4842, doi:10.1007/s11263-013-0620-5.

[36] Mark Everingham, Luc Van Gool, Christopher KI Williams, John Winn, and Andrew Zisserman. The pascal visual object classes (voc) challenge. International journal of computer vision, 88(2):303-338, 2010.

[37] Ross Girshick. Fast R-CNN. Proceedings of the IEEE International Conference on Computer Vision, 2015 Inter:1440-1448, 2015. arXiv:1504.08083, doi:10.1109/ICCV. 2015.169. 
[38] M. Everingham, L. Van Gool, C. K. I. Williams, J. Winn, and A. Zisserman. The PASCAL Visual Object Classes Challenge 2007 (VOC2007) Results. http://www.pascalnetwork.org/challenges/VOC/voc2007/workshop/index.html.

[39] M. Everingham, L. Van Gool, C. K. I. Williams, J. Winn, and A. Zisserman. The PASCAL Visual Object Classes Challenge 2010 (VOC2010) Results. http://www.pascalnetwork.org/challenges/VOC/voc2010/workshop/index.html.

[40] Tsung-Yi Lin, Michael Maire, Serge Belongie, James Hays, Pietro Perona, Deva Ramanan, Piotr Dollár, and C. Lawrence Zitnick. Microsoft coco: Common objects in context. In David Fleet, Tomas Pajdla, Bernt Schiele, and Tinne Tuytelaars, editors, Computer Vision ECCV 2014, pages 740-755, Cham, 2014. Springer International Publishing.

[41] Jifeng Dai, Yi Li, Kaiming He, and Jian Sun. R-fen: Object detection via region-based fully convolutional networks. In Advances in neural information processing systems, pages 379-387, 2016.

[42] Joseph Redmon, Santosh Divvala, Ross Girshick, and Ali Farhadi. You Only Look Once: Unified, Real-Time Object Detection. 2015. URL: http://arxiv.org/abs/1506. 02640, arXiv:1506.02640, doi:10.1109/CVPR.2016.91.

[43] Wei Liu, Dragomir Anguelov, Dumitru Erhan, Christian Szegedy, Scott Reed, Cheng-Yang $\mathrm{Fu}$, and Alexander C Berg. Ssd: Single shot multibox detector. In European conference on computer vision, pages 21-37. Springer, 2016.

[44] Joseph Redmon and Ali Farhadi. Yolov3: An incremental improvement. arXiv preprint arXiv:1804.02767, 2018.

[45] Tsung-Yi Lin, Priyal Goyal, Ross Girshick, Kaiming He, and Piotr Dollár. Focal loss for dense object detection. IEEE transactions on pattern analysis and machine intelligence, 2018.

[46] Tsung-Yi Lin, Piotr Dollár, Ross Girshick, Kaiming He, Bharath Hariharan, and Serge Belongie. Feature pyramid networks for object detection. In CVPR, volume 1, page 4, 2017.

[47] Cheng-Yang Fu, Wei Liu, Ananth Ranga, Ambrish Tyagi, and Alexander C Berg. Dssd: Deconvolutional single shot detector. arXiv preprint arXiv:1701.06659, 2017.

[48] Abhinav Shrivastava, Rahul Sukthankar, Jitendra Malik, and Abhinav Gupta. Beyond skip connections: Top-down modulation for object detection. arXiv preprint arXiv:1612.06851, 2016.

[49] Gianluigi Ciocca, Paolo Napoletano, and Raimondo Schettini. Food recognition: a new dataset, experiments and results. IEEE Journal of Biomedical and Health Informatics, 21(3):588-598, 2017. doi:10.1109/JBHI.2016.2636441.

[50] Han Xiao, Kashif Rasul, and Roland Vollgraf. Fashion-mnist: a novel image dataset for benchmarking machine learning algorithms, 2017. arXiv: cs.LG/1708.07747.

[51] Robert Geirhos, Patricia Rubisch, Claudio Michaelis, Matthias Bethge, Felix A Wichmann, and Wieland Brendel. Imagenet-trained cnns are biased towards texture; increasing shape bias improves accuracy and robustness. arXiv preprint arXiv:1811.12231, 2018. 
[52] Diederik Kingma and Jimmy Ba. Adam: a method for stochastic optimization (2014). arXiv preprint arXiv:1412.6980, 15, 2015.

[53] Léon Bottou. Large-scale machine learning with stochastic gradient descent. In Proceedings of COMPSTAT'2010, pages 177-186. Springer, 2010.

[54] Ashia C Wilson, Rebecca Roelofs, Mitchell Stern, Nati Srebro, and Benjamin Recht. The marginal value of adaptive gradient methods in machine learning. In Advances in Neural Information Processing Systems, pages 4148-4158, 2017. 